\title{
Local Effects Following Single and Repeat Intra- Articular Injections of Triamcinolone Acetonide Extended-Release: Results from Three Nonclinical Toxicity Studies in Dogs
}

\author{
Neil Bodick - Toni Williamson - Vibeke Strand - Becca Senter • \\ Scott Kelley $\cdot$ Rogely Boyce $\cdot$ Ruth Lightfoot-Dunn
}

Received: July 17, 2018 / Published online: September 10, 2018

(C) The Author(s) 2018

\section{ABSTRACT}

Introduction: Single intra-articular (IA) injections of poly(lactic-co-glycolic acid) (PLGA) microsphere-based triamcinolone acetonide extended-release (TA-ER; formerly FX006) demonstrated sustained, clinically relevant benefits in patients with knee osteoarthritis. The local effects of TA-ER were assessed in normal canine knees in three nonclinical studies.

Methods: Knees were evaluated for up to 6 weeks or 9 months after a single injection of TA-ER (2.1/6.25/18.75 mg TA), or TA crystalline

Enhanced Digital Features To view enhanced digital features for this article go to https://doi.org/10.6084/ m9.figshare.7016654.

Electronic supplementary material The online version of this article (https://doi.org/10.1007/s40744$018-0125-3)$ contains supplementary material, which is available to authorized users.

N. Bodick $(\bowtie) \cdot$ T. Williamson · B. Senter · S. Kelley Flexion Therapeutics, Inc., Burlington, MA, USA e-mail: nbodick@flexiontherapeutics.com

V. Strand

Division of Immunology/Rheumatology, Stanford

University School of Medicine, Palo Alto, CA, USA

R. Boyce

Beechy Ridge ToxPath LLC, Clay, WV, USA

R. Lightfoot-Dunn

Crucial Consulting, Inc., Westlake Village, CA, USA suspension (TAcs, $18.75 \mathrm{mg}$ TA), and for up to 6 months after three injections (every 1 or 3 months) of TA-ER (6.25/18.75 mg TA) or TAcs (18.75 mg). Vehicle-diluent, blank microspheres, and untreated knees were used as controls. Plasma and synovial fluid (SF) TA concentrations and standard histopathological assessment of the synovium were conducted. Articular cartilage morphology was assessed via modified Mankin scoring.

Results: Plasma and SF concentrations indicated prolonged dose-dependent TA joint residency with TA-ER compared with TAcs. Effects in articular cartilage were dose- and time-dependent and consistent with known effects of corticosteroids in the normal knee. Loss of Safranin $\mathrm{O}$ staining occurred, indicative of a reduction in cartilage matrix proteoglycan, and recovered in a similar manner for TA-ER and TAcs across all studies. Structural lesions were infrequent and generally comparable in severity between TA-ER and TAcs but slightly higher in incidence for TA-ER. Focal/multifocal foreignbody responses (FBR) to PLGA were observed in the superficial layer of the synovium, peaking after 4-6 weeks, with significant recovery or complete resolution by month 6 .

Conclusions: These findings suggest that the effects of IA injections of TA-ER on cartilage are predominantly transient, and comparable to those observed with TAcs in the normal canine knee joint. These mild effects in the normal joint differ from the beneficial effects observed 
with TA-ER and other corticosteroids in disease models. The synovial FBR to PLGA microspheres was focal and transient.

Funding: Flexion Therapeutics, Inc.

Plain Language Summary: Plain language summary available for this article.

Keywords: Biodegradable polymer drug carrier; Cartilage; Corticosteroid; Intra-articular; Microsphere; Triamcinolone acetonide extended-release

\section{PLAIN LANGUAGE SUMMARY}

A new form of steroid injection that stays in the joint for a longer period of time than older forms has been shown to work in knee osteoarthritis. The steroid in this new product is embedded in a biodegradable bead. As the bead biodegrades, the steroid is released into the knee. In order to see how this new product affects the knee joint, it was injected into the knees of dogs without osteoarthritis. The effects were like those seen in other studies with steroids injected into normal knees. Other studies have shown that steroids can cause damage to cartilage when injected into a normal knee, but in damaged knees (like in people with osteoarthritis), steroids are beneficial. The effects of the biodegradable beads were mild within the knee.

\section{INTRODUCTION}

Osteoarthritis (OA), the most common joint disorder in the United States, is recognized as a serious condition associated with increased mortality [1]. Inflammation plays a pivotal role in OA pathogenesis [2], providing a basis for the efficacy of traditional intra-articular (IA) corticosteroid (CS), such as triamcinolone acetonide crystalline suspension (TAcs), in relieving OA pain [3]. The systemic absorption of the drug from the joint within hours of injection [4] likely limits the duration of analgesic and antiinflammatory effects of IACS, and may also lead to systemic metabolic effects $[5,6]$. Despite the relatively short joint residence time, and due to high concentrations of traditional IACS within the joint space immediately postinjection, concerns have been raised regarding the potential for IACS to cause local cartilage damage [7]. Nonetheless, anti-inflammatory IACS remain a mainstay in symptomatic management of OA pain [3].

Triamcinolone acetonide extended-release (TA-ER; formerly known as FX006) is a novel, poly(lactic-co-glycolic acid) (PLGA) microsphere-based formulation of TA designed to release the drug slowly, over at least a 3-month period following IA injection. In phase 2 clinical pharmacokinetic (PK) studies, a single IA injection of $32 \mathrm{mg}$ TA-ER resulted in substantially longer TA joint residence time and lower systemic exposure [8], as well as lower postinjection blood glucose elevations compared with TAcs [9]. The differential PK/pharmacodynamic profile of TA-ER has translated into sustained, clinically relevant analgesic and functional benefits compared with saline-placebo and TAcs in phase 2/3 randomized controlled trials (RCTs) in patients with knee OA [10-12]. To date, nonclinical and clinical studies have reported protective effects of TA-ER on articular cartilage $[11,13]$ with a low incidence of minimal radiographic joint space narrowing in RCTs.

Safety studies in laboratory species such as rodents and Beagle dogs are an essential component of the evaluation of new therapeutics. Safety studies in animal models of disease are often impractical due to limited availability of the model, lack of historical data, necessity for prolonged administration, false-positive data, and importantly that the model may not closely mimic the human disease in terms of etiology and/or pathogenesis [14]. Therefore normal, healthy, well-characterized animal species are often utilized, but the limitations for predicting effects in the disease setting must be recognized. With respect to the evaluation of TA-ER, it is known that the response to corticosteroids in a normal synovial joint differs from responses in animal models of OA [13]. In healthy articular cartilage, chondrocytes are quiescent but engage in a low level of continual remodeling of the cartilage matrix to maintain normal structure and biochemical properties [15-17]. 
Corticosteroids perturb the balance of matrix resorption and synthesis, resulting in loss of Safranin O staining and fissuring of the articular cartilage surface [18]. In OA, articular chondrocytes release numerous cytokines and chemokines that result in enhanced cartilage matrix catabolism and degeneration, and in this setting, the clinical anti-inflammatory benefits of corticosteroids are observed [19-21].

Calculation of IA doses for animal safety studies is determined by scaling on the basis of synovial fluid (SF) volume, joint surface area, or on systemic drug exposure following IA injection; typically, IA doses and dosing intervals are designed to achieve SF drug concentrations at or greater than predicted to achieve therapeutic effects in patients. These factors must be considered when evaluating results in safety (toxicology) studies in healthy Beagle dogs with normal knees.

To further assess the local effects of and recovery from TA-ER injection(s), three singleor repeat-dose Good Laboratory Practice (GLP)compliant toxicology studies were conducted in Beagle dogs. In addition, PK modeling was performed to estimate TA concentrations in SF after multiple doses of TA-ER or TAcs.

\section{METHODS}

\section{Ethical Considerations}

Procedures were performed in accordance with regulations outlined in the US Department of Agriculture Animal Welfare Act [22] and in the Institute for Laboratory Animal Research's Guide for Care and Use of Laboratory Animals [23]. Study protocols were reviewed and approved by an institutional animal care and use committee.

Sample sizes and durations of monitoring for all studies were based on worldwide regulatory guidelines and are considered the minimum necessary to provide a preliminary assessment of tolerability and PK in dogs.

\section{Treatment}

All studies were conducted by Calvert Laboratories (Scott Township, PA). Animals were randomly assigned to treatment using the Path/Tox System (Version 1.7.1; Xybion Corporation, Cedar Knolls, NJ, USA) to achieve similar mean body weights between groups. Route of administration and doses of study treatments were selected based on those used in clinical trials, but dosing was limited by veterinary practice recommendations for the maximum reasonable IA injection volume in Beagle dogs $(1 \mathrm{ml})$. Treatments included: vehicle-diluent identical to that used in marketed TA-ER $(0.9 \%$ sodium chloride, $0.5 \%$ sodium carboxymethylcellulose, $0.1 \%$ polysorbate 80 ), blank microspheres (PLGA microspheres, 75:25 lactide:glycolide ratio with particle size range $20-100 \mu \mathrm{m}$ [median, $43 \pm 2 \mu \mathrm{m}$ for all lots]), TAcs (KENALOG ${ }^{\circledR}{ }_{-}$ 40 INJECTION [triamcinolone acetonide injectable suspension]; Bristol-Myers Squibb Company, Princeton, NJ, USA), and TA-ER (nominal drug load 1:3 [i.e., 25\% drug loading, $\mathrm{w} / \mathrm{w}]$ ). TA-ER and blank microspheres were prepared on the day of dosing by reconstituting individual vials with the appropriate volume of diluent to form a suspension containing the designated dose; TAcs was diluted in saline to obtain the appropriate concentration.

Animals were euthanized at time points described below by intravenous barbiturate overdose. To facilitate SF collection, $1 \mathrm{ml}$ of $0.9 \%$ saline was injected prior to removal of the joint fluid. To account for differences in SF sample dilution, the ratio of SF:blood urea nitrogen levels was used to adjust the TA concentrations detected in the diluted SF [24]. See the Online Supplement for more information on animal handling.

\section{Study Designs}

\section{Single-Dose Studies}

In the two single-dose studies, experimentally naive Beagle dogs (Marshall BioResources, North Rose, NY, USA) were randomly assigned to receive one of the following treatments in the right knee: vehicle-diluent; blank microspheres 
(75 mg PLGA); TAcs (18.75 mg TA); or low(2.1 mg TA, $8.33 \mathrm{mg}$ PLGA), mid- (6.25 mg TA, $25 \mathrm{mg}$ PLGA), or high- (18.75 mg TA, $75 \mathrm{mg}$ PLGA) dose TA-ER. The left knee served as an untreated control.

In the first study, ten animals of each sex aged 9-10 months were included in each treatment group and monitored for up to 6 weeks. In the second study, eight animals of each sex aged 10-13 months were included in each treatment group and monitored for up to 9 months. Blood samples for toxicokinetic analysis were collected from the jugular vein of three animals/sex/group in each of the groups receiving TA-ER or TAcs. In addition, SF samples were collected at necropsy from the injected and contralateral control knees. In the 6-week study, subsets of four animals/sex/group were euthanized on day 4 and week 4 , and two animals/sex/group at week 6 (Fig. 1). In the 9-month study, subsets of two animals/sex/group were sacrificed at month 3 , month 4, month 6, and month 9 (Fig. 1).

\section{Repeat-Dose Study}

In the third study, experimentally naive Beagle dogs (Marshall BioResources, North Rose, NY, USA), aged 12-14 months received three doses of assigned study treatment administered at two different dosing intervals. The first set of animals received injections every 3 months (Q3M) to explore the effects of repeat doses at an interval consistent with the anticipated minimum period of efficacy. The second set of animals received injections every month $(\mathrm{Q} 1 \mathrm{M})$ to explore the effects associated with a more intensive dosing regimen. Six animals/sex/group were randomly assigned to one of the treatment groups listed in Table 1.

Blood samples were collected from the jugular veins of three animals/sex/group between hour 1 and 60 days after each dose, and up to 6 months after the final dose. In addition, SF samples were collected from both knees at necropsy.

Subsets of three animals/sex/group were sacrificed at 1 month after the last dose (month 1) and 6 months after the last dose (month 6) for both Q1M and Q3M dosing regimens (Fig. 2).

\section{Assessments}

\section{Toxicokinetics}

Plasma samples were assessed for TA concentrations using validated liquid chromatography/tandem mass spectrometry (LC-MS/MS) methods. Samples of SF were evaluated for TA concentrations using the same LC-MS/MS methods, although the method has not been

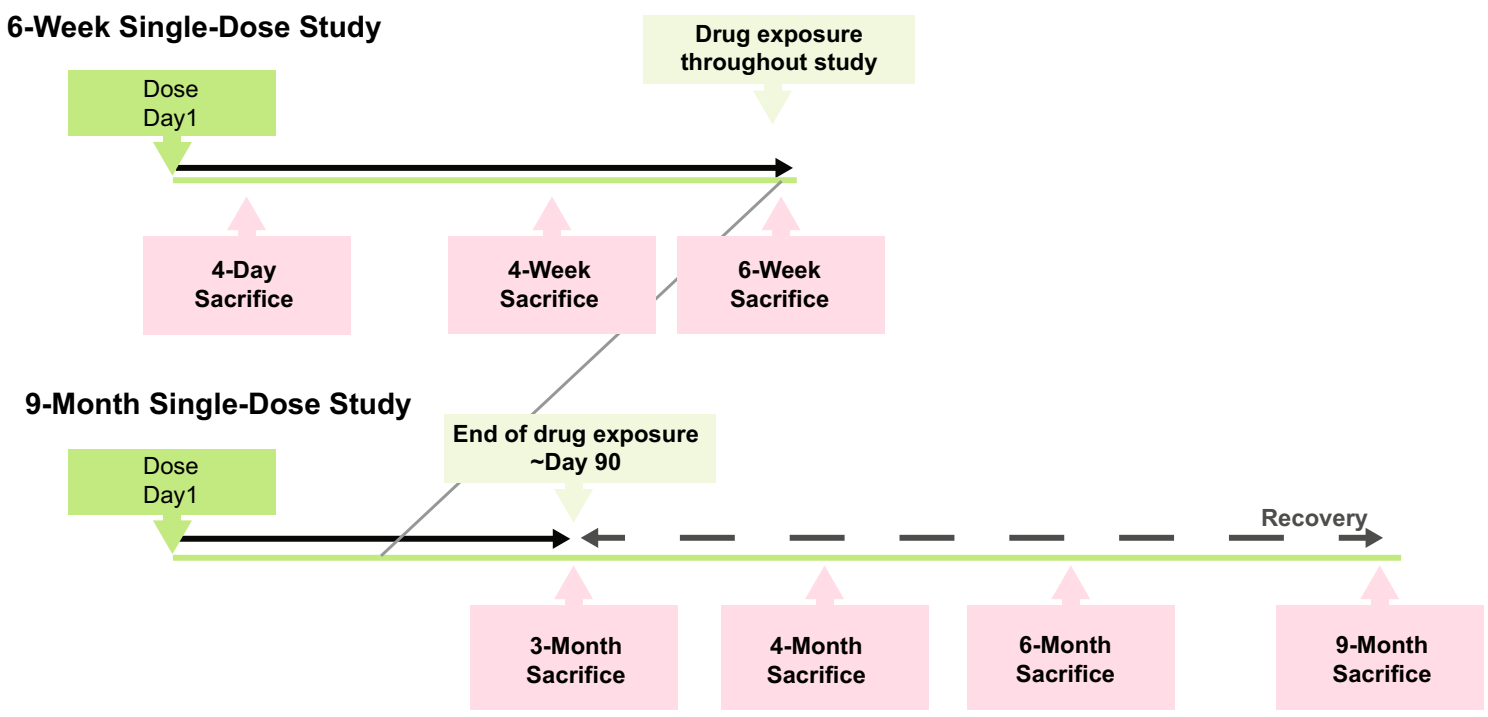

Fig. 1 Designs of single-dose 6-week (a) and 9-month (b) studies 


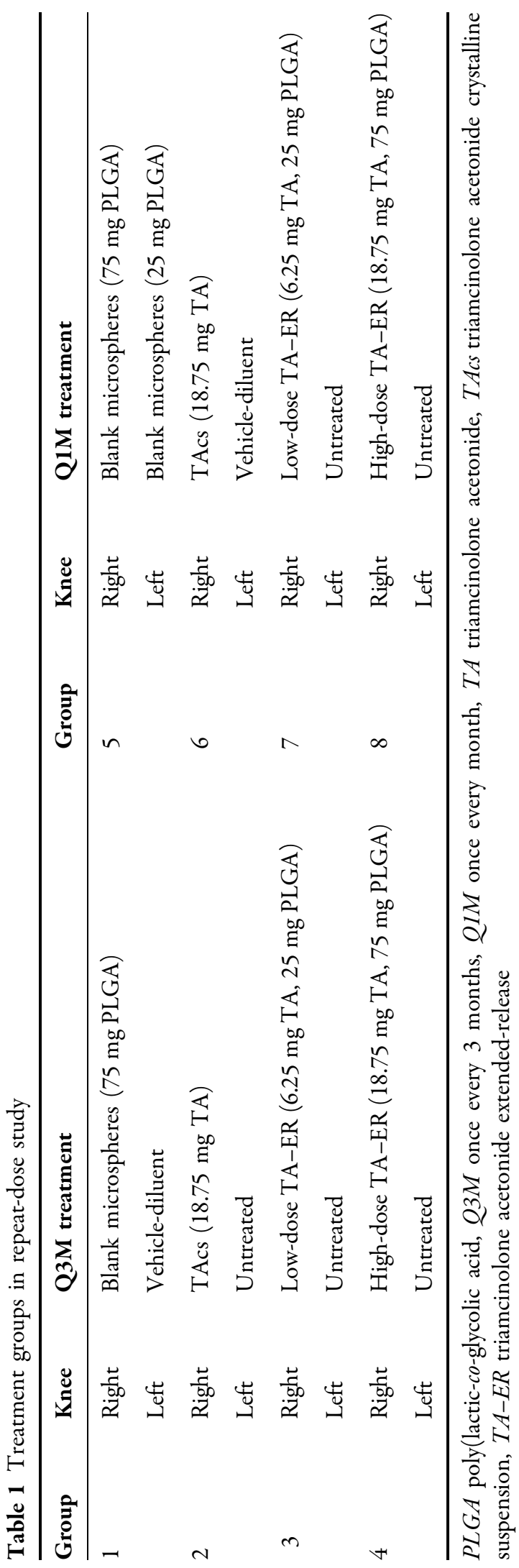

validated specifically for measurement of TA in SF. See the Online Supplement for more information on blood and SF sample collection and LC-MS/MS methods.

\section{Pharmacokinetic Modeling}

Data from the 6-week single-dose study was used to develop a PK model to characterize TA-ER and TAcs exposure in SF and plasma. Different compartmental PK models were tested during the development of the base model. Akaike information criterion, Bayesian information criterion, and the goodness-of-fit plots were used to compare potential candidate models and to choose the final model. The selected PK model was used to perform simulations and estimate plasma and SF TA concentrations after repeat doses of TA-ER and TAcs with Q1M and Q3M dosing. These simulations were also used to assess the duration for which the TA levels were maintained above a range of target effective concentrations $(10,30$, and $100 \mathrm{ng} / \mathrm{ml}$ ) in SF based on relative receptor potency, SF protein binding, and SF protein levels in OA patients $[25,26]$. See the Online Supplement for more information on PK model development and simulations.

\section{Histopathology}

Bone and cartilage from the medial femoral condyle, medial tibial plateau, and articular surface of the patella from each knee joint underwent decalcification, were embedded in paraffin, and sectioned with one section stained with hematoxylin and eosin (H\&E) stain and a second section stained with Safranin O. Knee joint synovial tissue was sectioned and stained with H\&E. See the Online Supplement for additional information on histologic procedures.

The presence of microspheres in tissues was inferred from the presence of characteristic variable-sized, round vacuoles (microspheres were removed/dissolved during the routine processing of specimens). A scoring system based on Mankin et al. [27] was used to evaluate local effects of TA-ER and TAcs on articular cartilage morphology for the tibial plateau, femoral condyle, and patella (Table S1). The 
Q3M

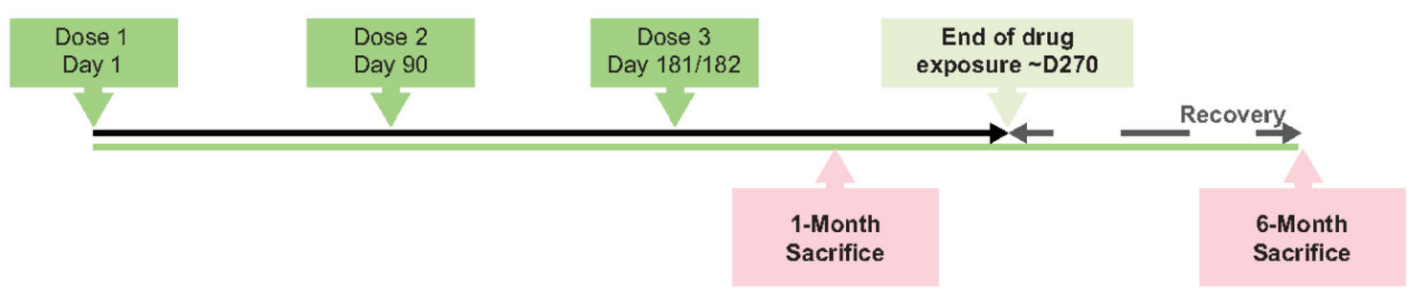

Q1M

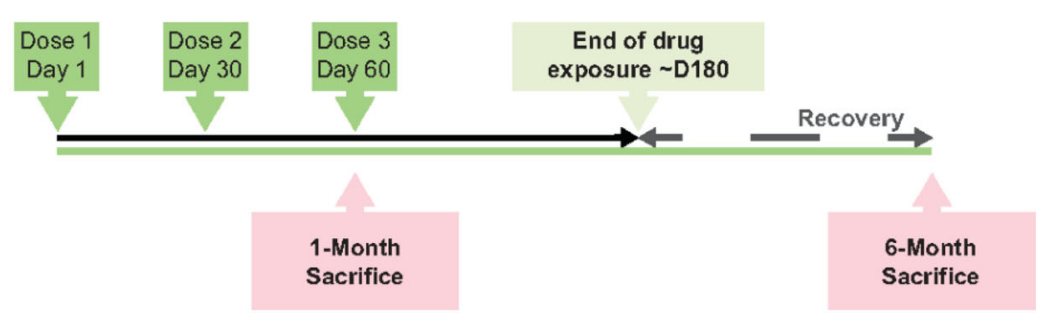

Fig. 2 Design of repeat-dose study. $D$ day, $Q 1 M$ once every month, $Q 3 M$ once every 3 months

modified Mankin scoring system assessed tidemark integrity by assessing whether blood vessels were seen traversing this area of the cartilage.

\section{Data Analysis}

For each study, plasma and SF TA concentration data were summarized by treatment group using descriptive statistics. Within each group, male and female animals exhibited similar plasma TA profiles, allowing data to be pooled across sexes. Toxicokinetic parameters derived from plasma TA concentration data included maximum plasma concentrations $\left(C_{\max }\right)$; area under the plasma concentration-time curves from hours 0 to $24 \mathrm{~h}\left(\mathrm{AUC}_{0-24}\right)$, to the last quantifiable concentration $\left(\mathrm{AUC}_{0-t}\right)$, and to infinity $\left(\mathrm{AUC}_{0-\infty}\right)$; and mean residence time in days from $0 \mathrm{~h}$ to infinity $\left(\mathrm{MRT}_{0-\infty}\right)$. Exploratory graphical analysis, modeling, and simulation of the PK data was conducted using validated PK data analysis software (Phoenix WinNonlin version 8.0, Certara Inc., Princeton, NJ, USA) by KinderPharm LLC (Exton, PA, USA). See the Online Supplement for additional information on statistical analysis.

Findings derived from evaluation of cartilage using modified Mankin scoring are presented for individual animals and specific joint sites rather than as mean Mankin scores per group to facilitate interpretation and illustrate observed variations. Individual animal Safranin $\mathrm{O}$ and structure scores from tibial plateau, distal femur, and patella were imported into data analytics software (TIBCO Spotfire version 7.6, TIBCO Software Inc., Palo Alto, CA, USA) for comparative analysis.

\section{RESULTS}

\section{Toxicokinetics}

In the 6-week study, systemic exposures $\left(C_{\max }\right.$ and AUC) were approximately dose-proportional across TA-ER doses (Fig. 3a). Absorption of TA from the knee joint was slower with TA-ER than with TAcs, resulting in lower systemic TA exposure at matched doses $(18.75 \mathrm{mg}$ TA) for high-dose TA-ER compared with TAcs (Fig. 3b). Plasma TA concentrations in the TAcs group were below the lower limit of quantitation (LLOQ; $0.1 \mathrm{ng} / \mathrm{ml}$ ) by 2 weeks postinjection. Plasma TA concentrations following highdose TA-ER remained above LLOQ at the end of the 6-week study. The $\mathrm{MRT}_{0-\infty}$ in SF with highdose TA-ER was approximately tenfold longer than with TAcs, although SF TA concentrations 
a

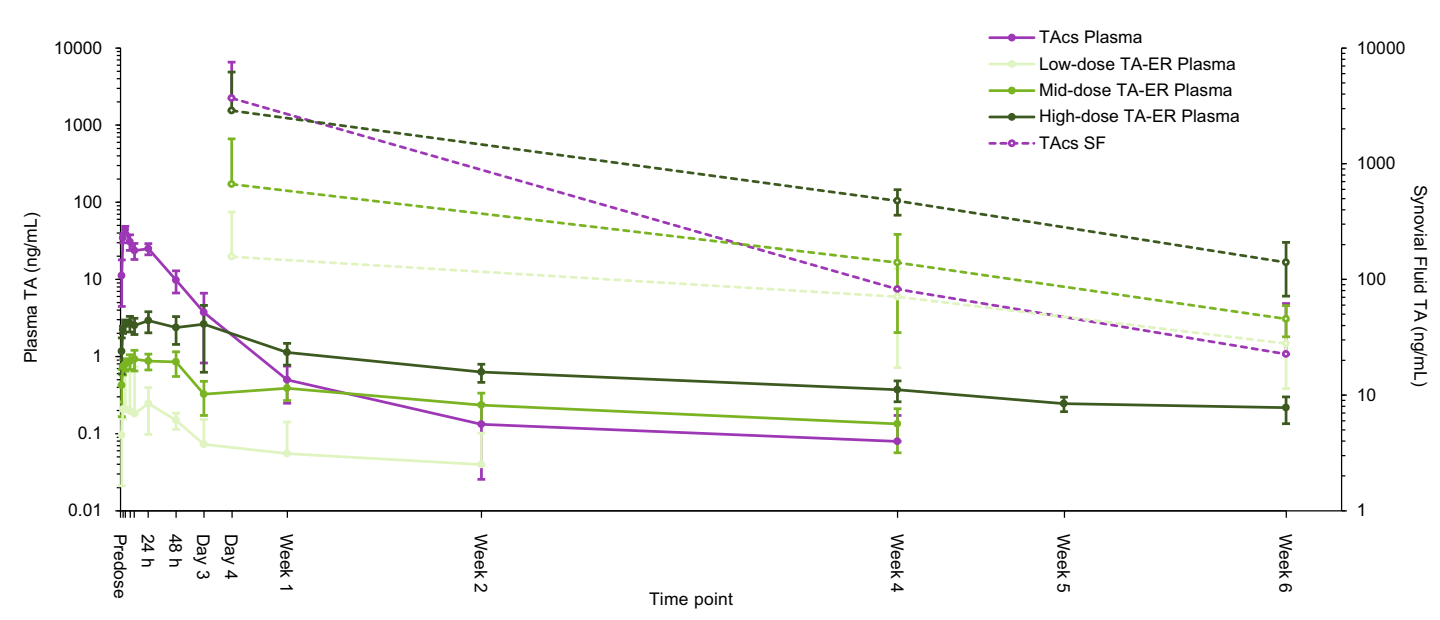

b

\begin{tabular}{l|cccc}
\hline & \multicolumn{4}{|c}{ Parameter, mean \pm SD } \\
& TAcs & $\begin{array}{c}\text { Low-dose } \\
\text { TA-ER }\end{array}$ & $\begin{array}{c}\text { Mid-dose } \\
\text { TA-ER }\end{array}$ & $\begin{array}{c}\text { High-dose } \\
\text { TA-ER }\end{array}$ \\
\hline Dose & & & & \\
TA & 18.75 & 2.1 & 6.25 & 18.75 \\
Microspheres & 0 & 8.33 & 25 & 75 \\
\hline $\mathrm{C}_{\text {max }}(\mathrm{ng} / \mathrm{mL})$ & $44.2 \pm 4.37$ & $0.282 \pm 0.129$ & $1.04 \pm 0.22$ & $3.09 \pm 0.76$ \\
$\mathrm{AUC}_{0-24}(\mathrm{ng} \cdot$ day $/ \mathrm{mL})$ & $27.4 \pm 3.72$ & $0.197 \pm 0.059$ & $0.834 \pm 0.161$ & $2.55 \pm 0.53$ \\
$\mathrm{AUC}_{0-\infty}(\mathrm{ng} \cdot$ day $/ \mathrm{mL})$ & $67.57 \pm 11.34$ & $3.211 \pm 2.105$ & $8.799 \pm 2.544$ & $31.80 \pm 7.03$ \\
$\mathrm{MRT}_{0-\infty}($ days $)$ & $1.5 \pm 0.25$ & $18.5 \pm 15.6$ & $9.8 \pm 4.6$ & $15.8 \pm 4.1$
\end{tabular}

Fig. 3 Plasma and synovial fluid concentrations (a) and plasma pharmacokinetic parameters (b) with TA. Note: data points below LLOQ not included. $A U C$ area under the curve, $C_{\max }$ maximum concentration, $I A$ intra-

were highly variable across all active treatment groups. Although high-dose TA-ER and TAcs resulted in similar SF TA concentrations at day 4 , higher concentrations were observed at weeks 4 and 6 with TA-ER versus TAcs (Fig. 3a).

Toxicokinetic data from the 9-month study confirmed the much lower systemic plasma exposure of TA-ER (Table S2). All SF samples contained either no detectable TA or concentrations below the LLOQ (data not shown), except for a single month 3 sample from a TAcstreated animal $(7.76 \mathrm{ng} / \mathrm{ml})$. articular, $L L O Q$ lower limit of quantitation, $M R T$ mean residence time, $S D$ standard deviation, $S F$ synovial fluid, TAcs triamcinolone acetonide crystalline suspension, $T A-E R$ triamcinolone acetonide extended-release

In the repeat-dose study, the relative bioavailability of TA in plasma for each postdosing period was like that observed in the single-dose studies, indicating the lack of systemic TA accumulation (Table S3).

\section{Pharmacokinetic Modeling}

A three-compartment PK model with first-order input and first-order elimination best described the simultaneous fit of both SF and plasma concentrations with both TA-ER and TAcs, 
where the initial faster release, followed by slower release of drug from SF to plasma, was well captured. Observed and predicted concentrations were overlaid side by side for both plasma and SF on linear and logarithmic scales, demonstrating an acceptable and unbiased fit of data to the model. Predicted concentrations of TA in SF were compared with TAcs and highdose TA-ER with both Q1M and Q3M regimens in the repeat-dose study. Based on relative receptor potency, SF protein binding, and SF protein levels in OA patients, the range of SF concentrations of TA required to maintain efficacy was between 6 and $100 \mathrm{ng} / \mathrm{ml}[25,26]$. The model predicted the duration of time TA concentrations would exceed $100 \mathrm{ng} / \mathrm{ml}$, the target $C_{\text {min }}$ required for efficacy. With Q3M dosing, observed and predicted SF concentrations with TAcs injections were less than the target $C_{\min }$ at approximately day 28 following each injection (Fig. 4a), but not until day 57 following highdose TA-ER, and were not less than $10 \mathrm{ng} / \mathrm{ml}$ with TA-ER at any point during the study (Fig. 4b). With Q1M dosing, the observed and predicted SF TA concentrations with TAcs dipped below the target $C_{\min }$ at day 28 after each monthly injection (Fig. 4c). However, the observed and predicted concentrations of TA in SF after all doses of TA-ER were significantly above the target $C_{\text {min }}$ over the entirety of the study (Fig. 4d).

\section{HISTOPATHOLOGY}

\section{Cartilage Effects and Mankin Scoring}

\section{Single-dose Studies}

Knees injected with a single dose of TAcs or TA-ER showed decreased Safranin O staining in articular cartilage, reflecting decreases in extracellular matrix glycosaminoglycan content (Fig. 5). Decreased glycosaminoglycan synthesis is a known effect of corticosteroids when administered directly into normal synovial joints in various animal models [28-31]. Loss of Safranin O staining within the middle, deep, and calcified zones of articular cartilage was variable but generally similar or slightly higher in mid- and/or high-dose TA-ER-treated knees compared with TAcs-treated knees through month 4 but comparable at months 6 and 9 (Fig. 6a, b). A few untreated knees also showed mild Safranin $\mathrm{O}$ staining loss, occurring between day 4 and week 6 . The contralateral injected knees were from either control or treated groups.

Structural effects in cartilage from the tibia or patella were sporadically observed in 1-2 knees (at one or two joint sites) for each TA-ER dose group from week 6 to month 9 and in one TAcs-treated knee (tibia) at month 4 (Table 2). These structural changes ranged in severity from surface irregularities to clefts to the tidemark region of the cartilage. Associated scores at these joint sites for loss of Safranin O staining were variable with scores of 1 (slight reduction, tangential zone) for tibia and ranging from 0 (normal) to 2 (moderate reduction [to tidemark]) for patella in joints treated with TAcs or TA-ER.

Cellularity scores in tibia or patella were typically associated with structural defects and scored as 2 (cellular cloning) or 3 (hypocellularity, necrosis). These effects are well-recognized responses to structural damage in articular cartilage and represent an attempted response to repair alterations in the cartilage matrix (data not shown) [32, 33].

\section{Repeat-Dose Study}

Q3M Safranin O Staining Loss of articular cartilage Safranin O staining occurred following Q3M dosing in untreated knees, in two knees treated with $75 \mathrm{mg}$ of blank microspheres, and in all TAcs- and TA-ER-treated knees at month 1. Loss of Safranin O staining at month 1 was comparable following TAcs and TA-ER injections, with a slightly lower incidence in lowdose TA-ER-treated knees. At month 6, loss of Safranin O staining was still present in all TAcsand TA-ER-treated knees, and one untreated knee, but showed significant recovery compared with month 1. Although the incidence of Safranin O staining loss was slightly higher in knees injected with TA-ER compared with TAcs, the severity was comparable (Fig. 6b).

Loss of Safranin $\mathrm{O}$ staining in untreated knees resulted from systemic exposure following injection of the contralateral knee with 

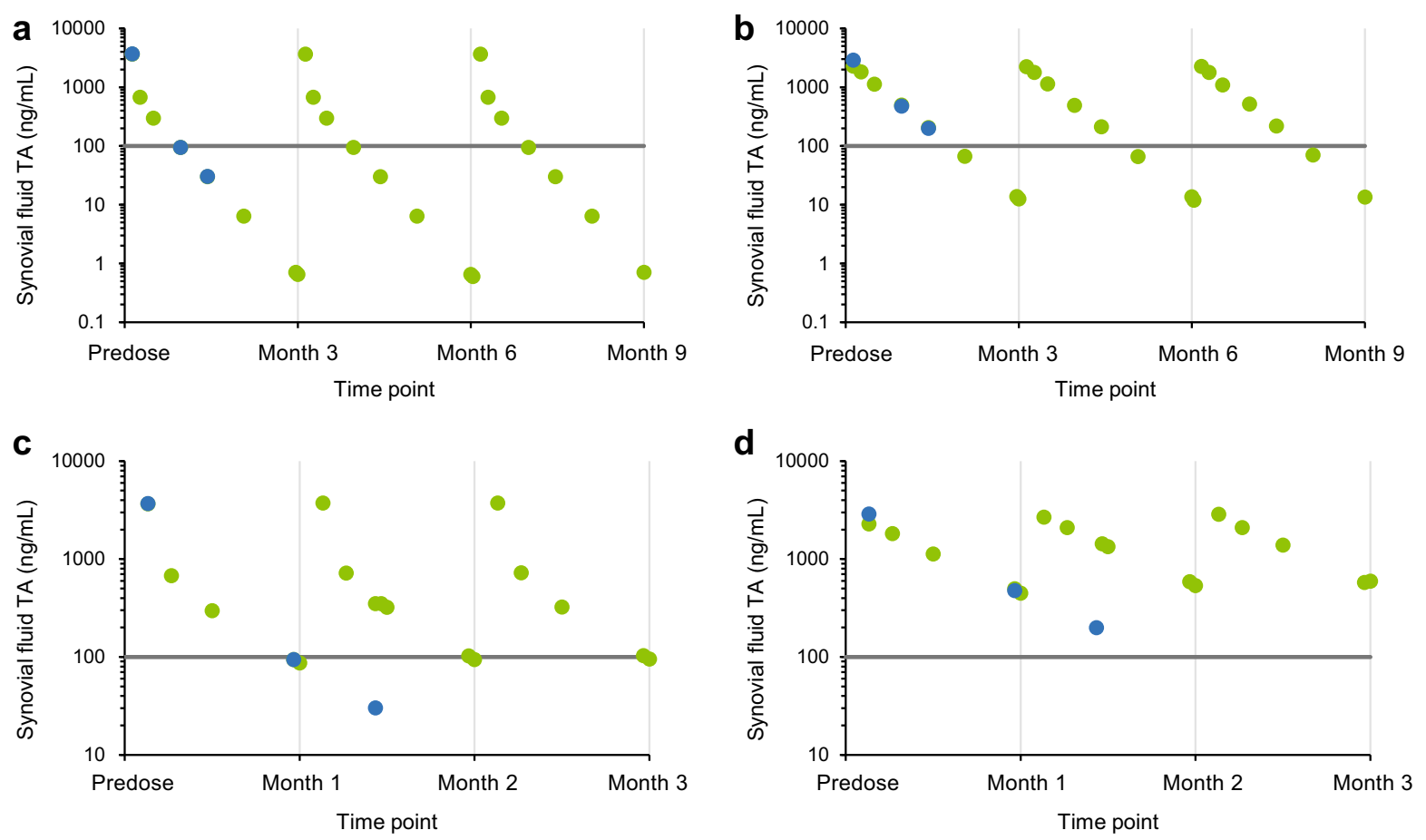

Fig. 4 Observed and modeled synovial fluid triamcinolone acetonide concentrations following single IA injections of TAcs modeled for Q3M administration (a), TA-ER modeled for Q3M administration (b), TAcs modeled for Q1M administration (c), and TA-ER modeled for Q1M administration $(\mathbf{d})$. Simulated data are represented as green

dots; observed data following single doses as blue dots. The horizontal line represents the target effective concentration, $C_{\min }(100 \mathrm{ng} / \mathrm{ml}) . T A$ triamcinolone acetonide, $T A c s$ triamcinolone acetonide crystalline suspension, $T A-E R$ triamcinolone acetonide extended-release

either TAcs or TA-ER. Recovery of Safranin O staining by month 6 in untreated knees reflected the shorter duration of exposure of the untreated knee to corticosteroid via systemic circulation compared with the longer joint residence time following TAcs and particularly TA-ER.

Q1M Safranin O Staining In knees dosed Q1M with TAcs or TA-ER, loss of Safranin O staining at months 1 and 6 was comparable (Fig. 6a). Month 6 scores demonstrated significant recovery compared with month 1 . Similar but less severe effects were observed in untreated knees following Q1M dosing, but also in knees treated with vehicle-diluent. All these animals experienced systemic exposure to TA (vehicle-diluent was administered into the left knee of animals receiving TAcs in the right knee.) As for Q3M dosing, loss of Safranin O

staining occurred in two knees injected with $75 \mathrm{mg}$ of blank microspheres at month 1 and in one knee at month 6 . This may be the result of the inherent variability of a subjective scoring system, but it is also possible that in an inflamed knee, local cytokine effects may cause an increase in cartilage matrix proteoglycan degradation $[19,20]$.

Q3M Structural Defects A few animals developed structural defects in articular cartilage at month 6 following Q3M dosing, but no structural defects were observed at month 1 except in two knees treated with $75 \mathrm{mg}$ of blank microspheres. In general, the femoral and tibial articular cartilage surfaces showed a higher incidence of structural effects than patellar cartilage, consistent with greater weight bearing of the tibial medial condyle and femur compared with patella [34]. Structure scores at month 6 
a

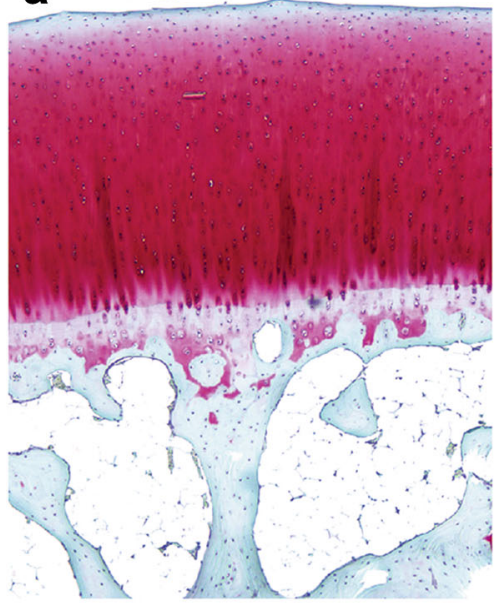

d

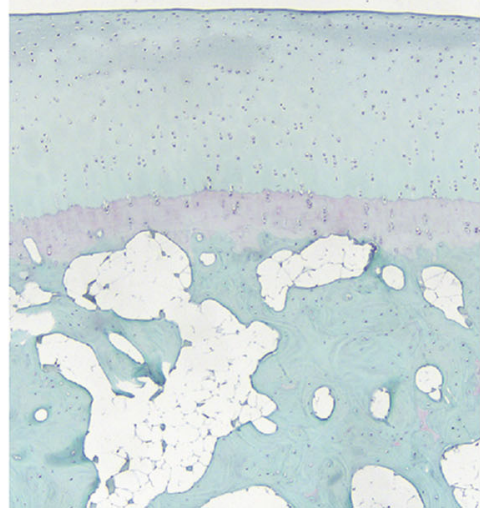

b

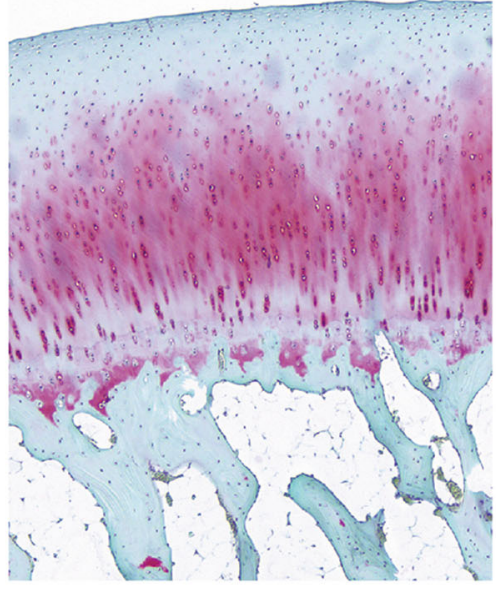

e

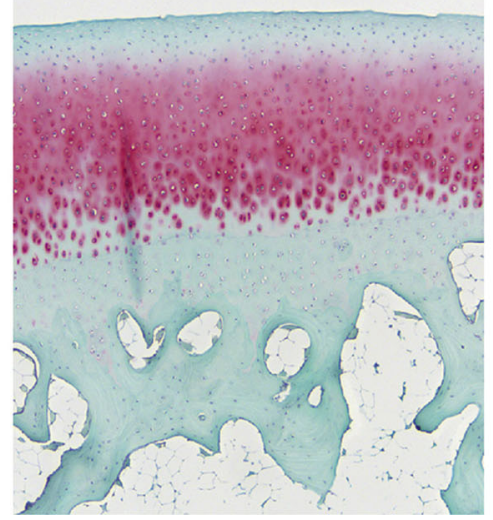

C

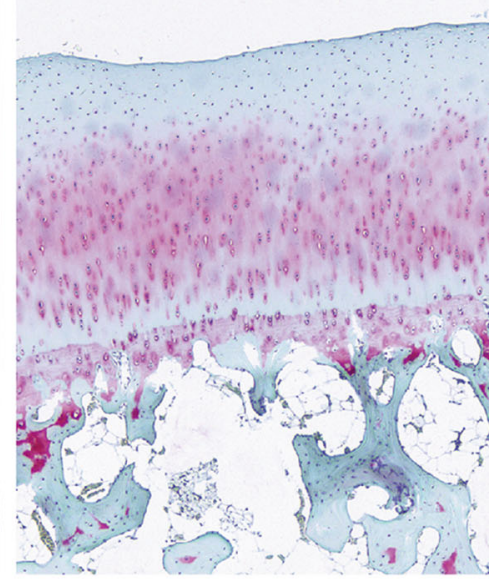

f

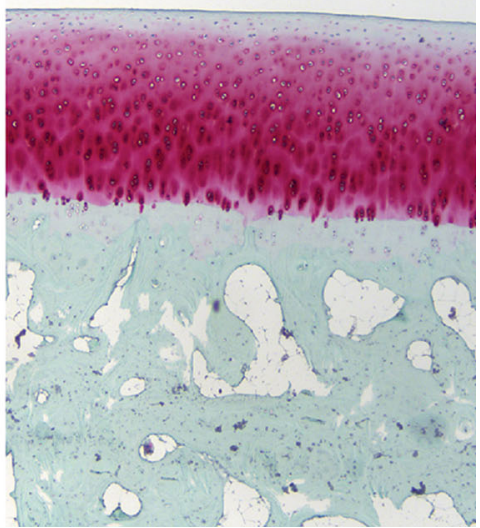

Fig. 5 Histologic evaluation of femoral cartilage in dogs at week 6 following single IA injections of vehicle-diluent (a), TAcs (b), high-dose TA-ER (c), and at month 4 (D), month 6 (e), and month 9 (f) following single IA injections of high-dose TA-ER (Safranin O staining, original magnification $\times 50$ ). Note: significant individual

typically ranged from 1 (surface irregularities) to 4 (clefts to the tidemark) with the following exceptions: 1 low-dose TA-ER-treated knee with a score of 5 (clefts to subchondral bone) in the patella, but other histopathologic changes (thickened joint capsule, thickened and frayed anterior cruciate ligament) suggest a pre-existing injury that contributed to joint destabilization and articular cartilage damage; one highdose TA-ER-treated knee with a score of 6 (complete disorganization) in femoral cartilage with a score of 1 for disruption of the tidemark. variability within treatment groups in Safranin O staining of femoral cartilage was observed. Sporadic scores for loss of Safranin O staining (score 1) in untreated knees are not shown in panels a or $\mathbf{b}$. TAcs triamcinolone acetonide crystalline suspension, $T A-E R$ triamcinolone acetonide extended-release

The highest combined structure score for all three joint surfaces was observed in a TAcstreated knee, also with combined loss of Safranin $\mathrm{O}$ staining scores significantly higher than any other knees at month 6 (Fig. 7a), leading to a Mankin score of 8 , the highest in the study.

Cellularity scores demonstrated a pattern similar to the single-dose studies and close correlations with structural defect scores (data not shown). Most scores were 1 (diffuse hypercellularity) or 2 (cloning) except for 1 TAcs Q3Mtreated knee with a score of 3 (hypocellularity, 

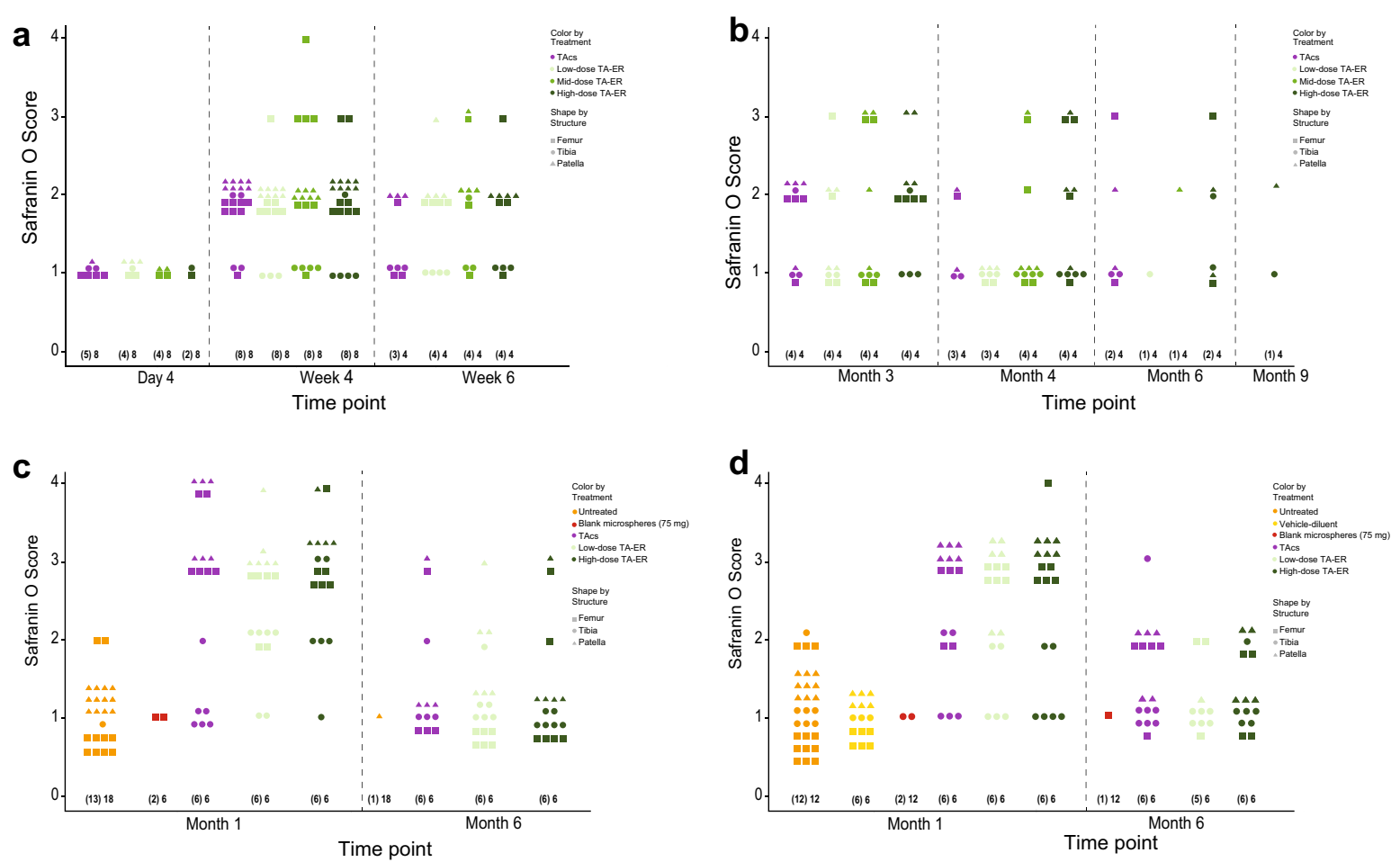

Fig. 6 Safranin $O$ staining scores for tibial plateau, femoral condyle, and patellar cartilage up to week 6 in the 6-week single-dose study (a), up to month 9 in the 9-month single-dose study (b), and at months 1 and 6 following Q3M administration (c) and following Q1M administration $(\mathbf{d})$ in the repeat-dose study. Treatments

are represented by colors, cartilage locations by shapes (see legend). Bold numbers represent the (number of knees with 1 or more Safranin O staining loss scores) number of knees evaluated per treatment. Scores of zero (0) are not included for clarity. TAcs triamcinolone acetonide crystalline suspension, $T A-E R$ triamcinolone acetonide extended-release

Table 2 Animals with Mankin structure scores after a single dose of TA-ER

\begin{tabular}{lllll}
\hline Timepoint (days) & Treatment & Structure scores & & Patella \\
\cline { 3 - 5 } & & Tibia & Femur & 0 \\
\hline 43 & Mid-dose TA-ER & 0 & 4 & 4 \\
& Untreated & 0 & 0 & 0 \\
& Low-dose TA-ER & 3 & 0 & 4 \\
& Mid-dose TA-ER & 0 & 0 & 4 \\
& Mid-dose TA-ER & 0 & 0 & 4 \\
\hline
\end{tabular}

$T A-E R$ triamcinolone acetonide extended-release

necrosis) in the femur at month 6 , and 1 lowdose TA-ER Q3M-treated knee with a score of 3 in the patella at the same time point.
Q1M Structural Defects At month 1, most structure scores were 1 (surface irregularities), except for 1 knee treated with $75 \mathrm{mg}$ of blank 


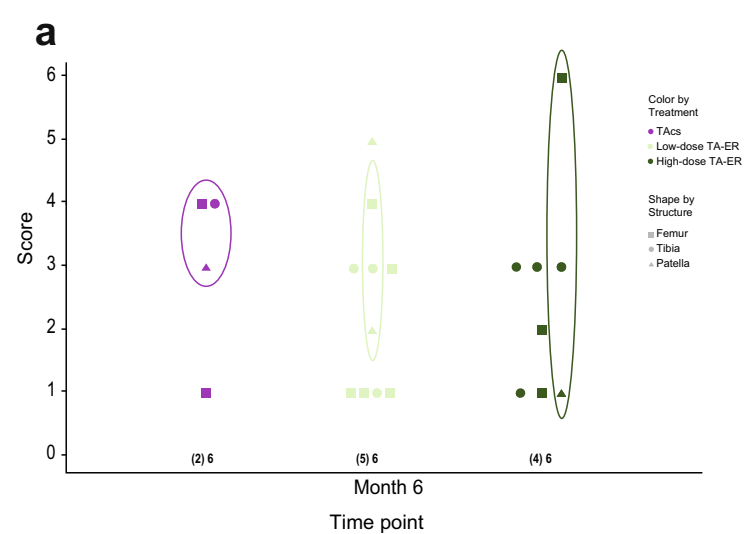

Fig. 7 Structure scores for femoral condyle and tibial plateau cartilage in the repeat-dose study at month 6 following Q3M administration (a) and at months 1 and 6 following Q1M (b). Treatments are represented by colors, cartilage locations by shapes (see legend). Bold numbers represent (number of knees with one or more structure scores) number of knees evaluated per treatment. Circles designate all structure scores for individual animals; one

microspheres with a score of 2 (pannus and surface irregularities) and one knee treated with high-dose TA-ER with a score of 3 (clefts to transitional zone). Structure scores of 0 were recorded for all TAcs-treated knees at month 1. At month 6, however, scores were comparable between TA-ER- and TAcs-treated knees (Fig. 7b). As in the Q3M dose groups, tibial and femoral cartilage were more frequently affected than patellar cartilage.

Comparison of Effects of TAcs and TA-ER on Cartilage Following Q3M and Q1M Dosing Following Q3M dosing, SF levels of TA were predicted to fall below the target $C_{\text {min }}$ during the 3-month interdose interval in all groups, although the drug cleared faster from TAcs-treated knees than high-dose TA-ER-treated knees (Fig. 4a, b). At month 1, loss of Safranin O staining was comparable with TAcs and TA-ER injections and no structural effects were observed. At month 6, Safranin O staining loss improved to a similar extent in both TAcsand TA-ER-treated knees but structural defects were observed with higher incidence in TA-ERtreated than TAcs-treated knees, although maximum severities were comparable. It is likely that the substantial cumulative exposure b

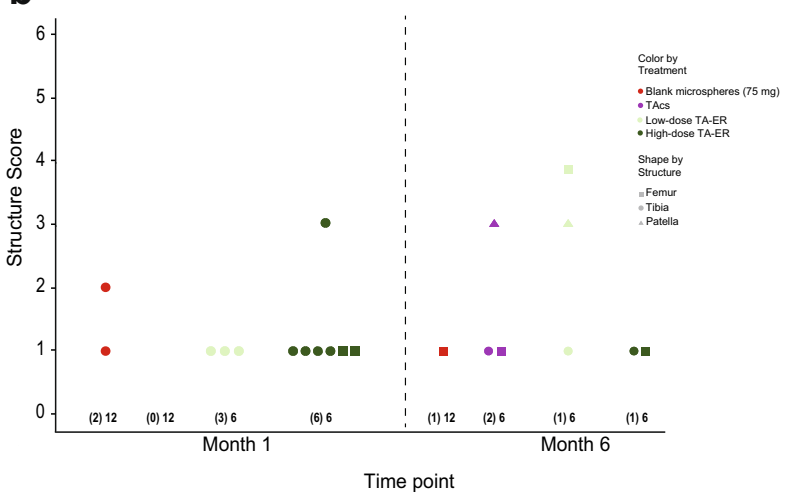

animal with the highest structure score for a single joint surface (high-dose TA-ER), one animal with structure scores likely arising from a pre-existing injury (low-dose TA-ER), and one animal with the highest combined structure score across all joint surfaces (TAcs). TAcs triamcinolone acetonide crystalline suspension, $T A-E R$ triamcinolone acetonide extended-release

of cartilage with TA-ER over almost 9 of the 12 months on study resulted in a higher incidence of structural effects. In one knee treated with TAcs with the highest Mankin score in the study (8.00), plasma TA concentrations after the first and last doses were substantially prolonged, remaining above levels observed in some highdose TA-ER animals. Structural lesions in this individual TAcs-treated animal were likely due to the longer exposure of cartilage to TA.

For Q1M dosing, the comparison between TAcs and TA-ER differs. At month 1, Safranin O scores were comparable between groups, but structural changes were observed only in TA-ER-treated knees with no clear relationship to dose, except perhaps the incidence. Concentrations of TA-ER were predicted to remain markedly above $100 \mathrm{ng} / \mathrm{ml}$ for the entire study period in high-dose TA-ER-treated knees (Fig. 4c, d), whereas SF TA concentrations fell to approximately $100 \mathrm{ng} / \mathrm{ml}$ during interdose intervals in TAcs-treated knees. At month 6, loss of Safranin O staining showed similar recovery in all TAcs- and TA-ER-treated knees and structure scores were comparable. TAcs-treated knees were predicted to clear drug faster from SF after the last dose compared with TA-ER-treated knees but nevertheless, drug exposure was 
sufficient in all groups to result in similar cartilage structure scores.

\section{Comparison of Effects of TA-ER on Cartilage} Between Q3M and Q1M Dosing Treatment with TA-ER Q1M resulted in structural lesions at month 1 but TA-ER Q3M resulted in no structural lesions at month 1 , even though Safranin O staining loss at month 1 was more pronounced with the Q3M regimen. Structural lesions likely resulted from the more intensive Q1M dosing schedule that maintained SF TA levels substantially above the target $C_{\min }$, but for Q3M dosing, allowed for recovery between doses. At month 6, a higher incidence and severity of structural lesions was observed for Q3M dosing than Q1M dosing, although some recovery in loss of Safranin $O$ staining was observed with both dosing regimens. Although there was more washout time between doses for TA-ER Q3M-treated knees, cumulative drug exposure in SF was sustained at or above the target $C_{\min }$ over 6 of 12 months for Q3M dosing versus 4 of 12 months in Q1M-treated knees.

\section{Foreign Body Response to PLGA Microspheres}

In the 6-week single-dose study, microspheres were present in SF samples at day 4, week 4, and week 6 . In the 9-month single-dose study, microspheres were present only at month 3 in a SF sample from a single knee injected with blank microspheres.

Similarly, in the repeat-dose study, microspheres and microsphere fragments were present in SF in 75-mg blank microsphere- and TA-ER-treated knees at month 1 in both the Q3M and Q1M regimens. By month 6, no microspheres were observed except for microsphere fragments in a few knees, suggesting near-complete degradation. Little-to-no TA-ER is expected to have remained in these microsphere fragments, consistent with undetectable levels of TA-ER in SF at month 6 in the 9-month single-dose study.

\section{Single-Dose Studies}

Histopathologic evaluations of the synovium showed a mild, focal or multifocal, reversible foreign body response (FBR) in blank microsphere- and TA-ER-treated knees (Table 3, Fig. 8). Microspheres were observed only within the knee joint and sequestered within or on the synovial membrane by clusters of type A synoviocytes (resident macrophages within the synovium). The FBR was evident from day 4 to month 4, most pronounced at week 4, and related to the dose of microspheres. Responses consisted principally of macrophage and multinucleated giant cell infiltration in association with microspheres in the synovium through month 4 (Fig. 8b, c), with recovery evident at month 6 (Table 3).

Synovial inflammatory cell infiltration, predominantly lymphocytes and plasma cells, was observed, but also occasionally included neutrophils and/or eosinophils. This effect was also occasionally observed in knees treated with vehicle-diluent and untreated knees, in the absence of a FBR. The inflammatory cell infiltrate was sporadic at weeks 4 and 6 in knees injected with blank microspheres, and in knees injected with blank microspheres or high-dose TA-ER at months 3, 4, 6, and 9 (Fig. 8e). Occurrence of this same finding with greater frequency but similar severity at month 9 in knees treated with TAcs in the absence of a FBR also suggests that this effect is not solely related to FBR (Tables S4 and S5).

Synovial hyperplasia occurred in response to the FBR and was also observed sporadically across all treatments at various time points (Tables S4 and S5). The frequency of synovial hyperplasia was greater in knees receiving blank microspheres or TA-ER in the presence of a clear FBR (Fig. 8b, c). Gradual resolution of these effects occurred with recovery of normal synovial morphology by week 6 (Fig. 8f, Tables S4 and S5).

Fibroplasia (activation and proliferation of fibroblasts) was a secondary response to the FBR and observed within the synovial intima in a few knees treated with blank microspheres (day 4) and in those treated with high-dose TA-ER (day 4 and week 4) but had completely resolved without fibrosis by week 6 . However, minimal to slight fibrosis was also evident in a few TA-ER-treated knees at months 3-6, but not month 9 (Tables S4 and S5). 


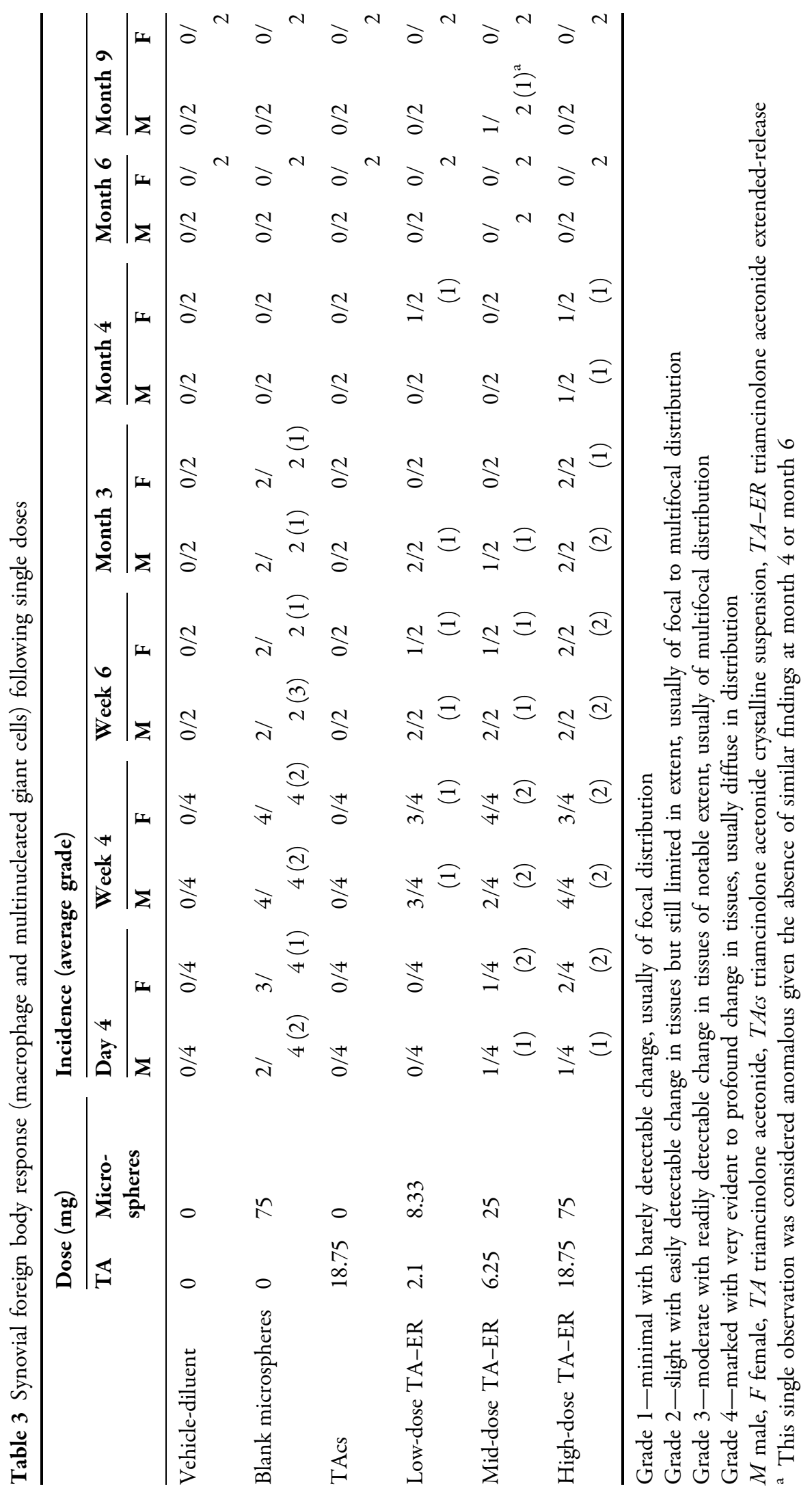



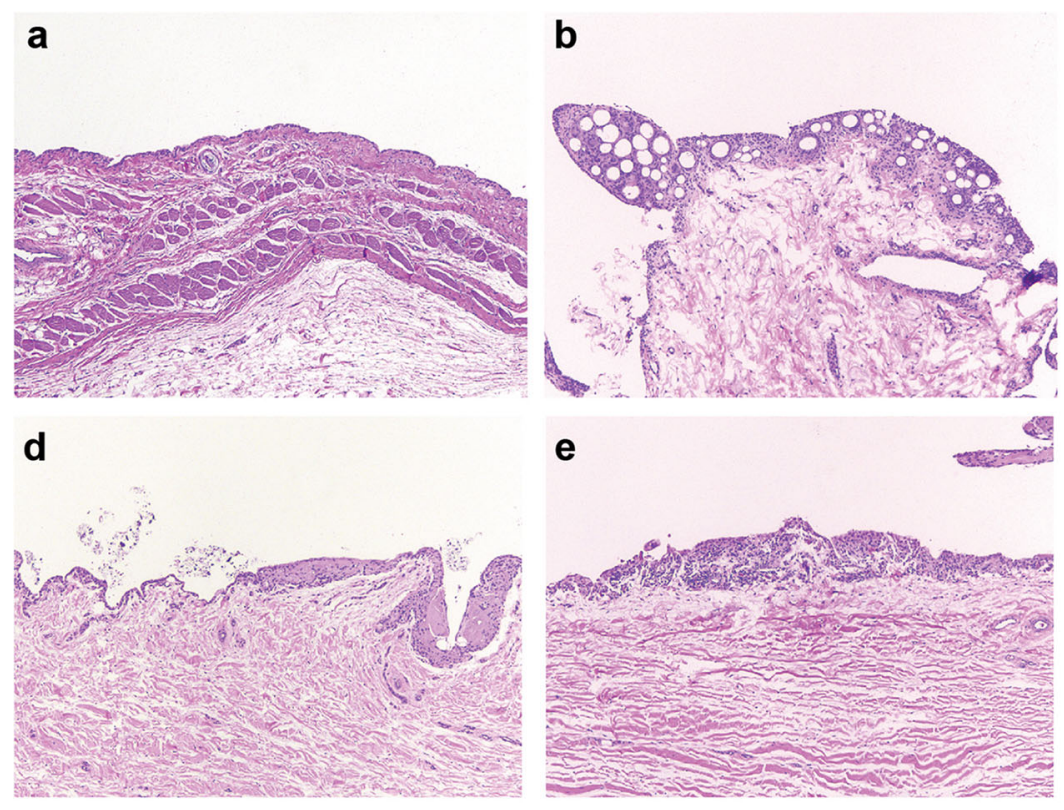

\section{C}

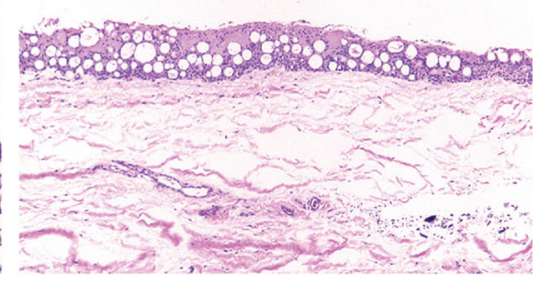

f

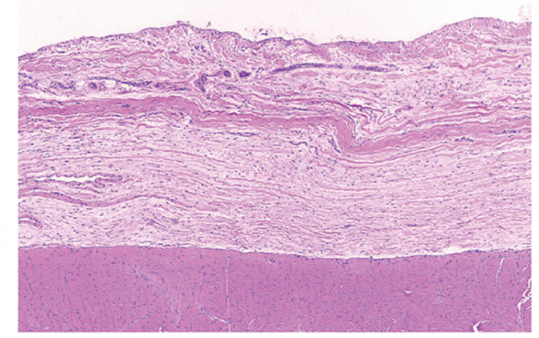

Fig. 8 Histologic evaluation of synovium from injected knee joints in dogs at week 4 following a single IA injection of vehicle-diluent (a), blank microspheres (b), high-dose TA-ER (c), and at month 3 (d), month 4 (e),

\section{Repeat-Dose Study}

In the repeat-dose study, PLGA-dependent FBR was present in some 75-mg blank microsphereand TA-ER-treated knees with both Q3M and Q1M dosing (Table 4). In general, the response was related to the dose of microspheres. As in the single-dose studies, the FBR was most pronounced at month 1 with increased severity for Q1M compared with Q3M dosing. At month 6, residual (minimal) effects were present in Q3Mdosed groups but complete resolution of the FBR occurred in the low-dose TA-ER and blank microsphere Q1M groups.

In contrast to single-dose studies, a synovial inflammatory cell infiltrate was clearly associated with the FBR at months 1 and 6 with both Q1M and Q3M dosing (Table S6). Although similar effects were observed in some vehicle-diluent-treated and untreated knees, the effect was more frequent and/or more pronounced in TA-ER- and 75-mg blank microsphere-treated knees. The most prominent effects, although only graded as slight, were present with Q1M dosing with no evidence of recovery from months 1 to 6 . and month $9(\mathbf{f})$ following single IA injections of high-dose TA-ER (hematoxylin and eosin staining, original magnification $\times 50) . T A-E R$ triamcinolone acetonide extendedrelease

An increased incidence of synovial hyperplasia was observed in some knees injected with TAcs or TA-ER, but also in some knees treated with vehicle-diluent or $75 \mathrm{mg}$ of blank microspheres. A clear relationship to treatment or dose was lacking (Table S6).

Synovial intimal fibrosis, predominantly minimal and focal, was associated with 75-mg blank microsphere and TA-ER treatment and is an expected consequence of a chronic FBR evident with both Q1M and Q3M dose regimens. Synovial fibrosis was more frequent, but of similar severity, in knees treated with $75 \mathrm{mg}$ of blank microspheres compared with $25 \mathrm{mg}$ of blank microspheres. Similarly, the incidence was slightly higher in knees treated with high-dose TA-ER compared with low-dose TA-ER-treated knees, reflecting the higher dose of microspheres.

Other infrequent findings (synovial neovascularization, acellular debris on the synovial surface) were associated predominantly with more pronounced FBR, and others (synovial focal degeneration/granulation, synovial mineralization) were associated with TAcs and TA-ER administration (data not shown). 
Table 4 Synovial foreign body response (macrophage and multinucleated giant cells) following repeat doses

\begin{tabular}{|c|c|c|c|c|c|c|c|c|c|c|}
\hline & \multirow{2}{*}{\multicolumn{2}{|c|}{ Dose (mg) }} & \multicolumn{8}{|c|}{ Incidence (average grade) } \\
\hline & & & \multicolumn{4}{|l|}{$\overline{Q 3 M}$} & \multicolumn{4}{|l|}{ Q1M } \\
\hline & \multirow[t]{2}{*}{$\overline{T A}$} & \multirow{2}{*}{$\begin{array}{l}\text { Micro- } \\
\text { spheres }\end{array}$} & \multicolumn{2}{|c|}{ Month 1} & \multicolumn{2}{|c|}{ Month 6} & \multicolumn{2}{|c|}{ Month 1} & \multicolumn{2}{|c|}{ Month 6} \\
\hline & & & $\bar{M}$ & $\mathbf{F}$ & $\overline{\mathbf{M}}$ & $\mathbf{F}$ & $\bar{M}$ & $\mathbf{F}$ & $\overline{\mathbf{M}}$ & $\mathbf{F}$ \\
\hline Vehicle-diluent & 0 & 0 & $0 / 3$ & $0 / 3$ & $0 / 3$ & $0 / 3$ & $0 / 3$ & $0 / 3$ & $0 / 3$ & $0 / 3$ \\
\hline Blank microspheres ( $25 \mathrm{mg})$ & 0 & 25 & - & - & - & - & $\begin{array}{l}3 / \\
3(2)\end{array}$ & $\begin{array}{l}3 / \\
3(2)\end{array}$ & $0 / 3$ & $0 / 3$ \\
\hline Blank microspheres $(75 \mathrm{mg})$ & 0 & 75 & $\begin{array}{l}3 / \\
\quad 3(2)\end{array}$ & $\begin{array}{l}3 / \\
3(3)\end{array}$ & $0 / 3$ & $\begin{array}{l}2 / \\
\quad 3(1)\end{array}$ & $\begin{array}{l}3 / 3 \\
(3)\end{array}$ & $\begin{array}{l}3 / 3 \\
(3)\end{array}$ & $0 / 3$ & $\begin{array}{l}1 / \\
3(1)\end{array}$ \\
\hline TAcs & 18.75 & 0 & $0 / 3$ & $0 / 3$ & $0 / 3$ & $\begin{array}{l}1 / 3 \\
(1)\end{array}$ & $0 / 3$ & $0 / 3$ & $0 / 3$ & $0 / 3$ \\
\hline Low-dose TA-ER & 6.25 & 25 & $\begin{array}{l}2 / 3 \\
\quad(2)\end{array}$ & $\begin{array}{r}3 / 3 \\
(1)\end{array}$ & $0 / 3$ & $\begin{array}{r}1 / 3 \\
(1)\end{array}$ & $\begin{array}{r}3 / 3 \\
(3)\end{array}$ & $\begin{array}{r}3 / 3 \\
(1)\end{array}$ & $0 / 3$ & $0 / 3$ \\
\hline High-dose TA-ER & 18.75 & 75 & $\begin{array}{l}3 / 3 \\
(2)\end{array}$ & $\begin{array}{l}3 / 3 \\
(2)\end{array}$ & $\begin{array}{l}1 / \\
3(1)\end{array}$ & $\begin{array}{l}2 / 3 \\
(1)\end{array}$ & $\begin{array}{l}3 / 3 \\
(3)\end{array}$ & $\begin{array}{l}3 / 3 \\
(2)\end{array}$ & $\begin{array}{l}1 / \\
3(1)\end{array}$ & $\begin{array}{l}3 / 3 \\
(1)\end{array}$ \\
\hline
\end{tabular}

Grade 1-minimal with barely detectable change, usually of focal distribution

Grade 2-slight with easily detectable change in tissues but still limited in extent, usually of focal to multifocal distribution Grade 3-moderate with readily detectable change in tissues of notable extent, usually of multifocal distribution

Grade 4-marked with very evident to profound change in tissues, usually diffuse in distribution

$M$ male, $F$ female, $T A$ triamcinolone acetonide, $T A c s$ triamcinolone acetonide crystalline suspension, $T A-E R$ triamcinolone acetonide extended-release

\section{DISCUSSION}

In three GLP-compliant dog toxicity studies, the local effects of IA injection(s) of an extendedrelease corticosteroid (TA-ER) were examined at doses ranging from 0.22 to 1.95 times the FDAapproved 32-mg human dose based on calculation of human equivalent dose. The doses used also reflected multiples of the approved human dose ranging from 0.24 to 7.61 based on either comparison of joint surface area or SF concentration (Table S7). Overall, IA injections of TA-ER demonstrated typical corticosteroid-related effects in normal knee joints that were consistent with the product's mechanism of release and like those observed with traditional TAcs used for treatment of OA. PLGA microspheres resulted in a focal FBR which was reversible, except for some mild secondary effects.

\section{Toxicokinetics}

The toxicokinetic results of the single-dose studies are consistent with those of a phase $2 \mathrm{PK}$ analysis conducted in humans with knee OA [8]. In that study, TA concentrations in SF following a single TAcs injection were quantifiable at week 6 in only two of eight patients (geometric mean, $7.7 \mathrm{pg} / \mathrm{ml}$ ), whereas TA was still quantifiable at week 12 in seven of nine patients (geometric mean, $290.6 \mathrm{pg} / \mathrm{ml}$ ) following a single TA-ER injection. Furthermore, the plasma concentration profile of TA-ER indicated much slower absorption of TA into the circulation with TA-ER than TAcs. This ability of TA-ER to remain within the joint as it releases $\mathrm{TA}$ is attributable to the size of the microspheres (median $=43 \pm 2 \mu \mathrm{m} ;$ range $2-100 \mu \mathrm{m}$ ) and local sequestration by type 1 synoviocytes $[35,36]$. 


\section{Cartilage Effects and Mankin Scoring}

The effects of IA corticosteroid injections on normal articular cartilage are well understood. For in vivo studies of animals without joint inflammation of joint inflammation or instability, IACS leads to progressive cartilage matrix degeneration that is dose- and duration-dependent [18, 37]. The earliest effect recorded according to the Mankin scoring system is a reversible loss of Safranin O staining (representing loss of cartilage matrix proteoglycan) accompanied by loss of proteoglycan-bound water molecules and cations. Decreased hydration of articular cartilage can contribute to development of cartilage structural defects such as clefts to the tidemark and remodeling of subchondral bone. Susceptibility of knee articular cartilage to these effects can vary locally, presumably due to the differential loading of the joint surfaces [38, 39]. Minor structural alterations restricted to the superficial zone of the cartilage may also have the potential to repair via intrinsic mechanisms $[33,40]$.

A modified Mankin scoring system was used to evaluate and grade the effects of TA-ER and TAcs on articular cartilage in these studies. This scoring system has been widely used in the evaluation of OA in humans and disease models. Recently, newer modified scoring systems have been proposed [41, 42].

The use of the Mankin scoring has some limitations in these studies since, in general, group sizes are small $(n=2-6)$ per sex, per time point, and treatment group. This is challenging when time to development and resolution of different effects in cartilage varies. Early development of loss of Safranin O staining was followed by structural and cellular effects at later time points, but it is challenging to draw clear correlations between Safranin O staining loss and structural defects for all time points, treatments, and areas of the articular surfaces of the joint. Other factors influencing cartilage matrix integrity and resilience are also likely to have been perturbed but were not measured (e.g., degradation of collagen). Loss of Safranin O staining occurs diffusely throughout articular cartilage, but structural defects are focal. In this study, only one section of cartilage at each joint site was evaluated and so the risk posed by loss of Safranin O staining may not be accurately reflected in the recorded focal structural lesions. This is a point addressed in more recent scoring systems with a recommendation to evaluate more sections per joint in addition to scoring the surface extent of structural changes [42].

The tidemark is the basophilic interface between calcified and noncalcified cartilage, and newly developed blood vessels (neovascularization) across the tidemark can be indicative of the initiation of a reparative response to injury [28]. A tidemark integrity score of 1 was recorded in three knee joints treated with low- or high-dose TA-ER at month 6 with Q3M dosing. Two of the three scores appear likely to be anomalous since they are associated with structural scores of 1 (surface irregularities) in a low-dose TA-ERtreated knee, and a structural score of 2 (pannus and structural irregularities) in a high-dose TA-ER-treated knee. The absence of cartilage clefts in these joints suggests that the vascular channels across the tidemark may have been a normal anatomical structure which has previously been described in normal joints [43, 44]. In the remaining high-dose TA-ER-treated knee with a tidemark integrity score of 1 , this result seems highly plausible, given the presence of a severe structural defect in the femur (score of 6 , complete disorganization). More recent scoring systems, such as the Osteoarthritis Research Society International (OARSI) cartilage histopathology grade assessment, focus more extensively on the pathology of subchondral bone and attempt to ensure that evaluation of all endpoints and lesions is integrated [42].

In these three dog studies, group mean Mankin scores for any treatment group at a specific time point varied between 0 and 3.5 out of a maximum of 14 (Table S8) indicating generally mild effects on cartilage with either TA-ER or TAcs. This also illustrates the recognized challenge of discriminating accurately between treatments when Mankin scores are low [45]. Therefore, we believe the use of visual graphic systems such as Spotfire to illustrate individual data points allows for meaningful interpretation of complex datasets in addition to composite Mankin scores for individual joints and treatments. 
Overall, there was a poor correlation between Safranin $\mathrm{O}$ scores and structural lesions, since more severe structural lesions may not repair, yet concurrent Safranin O scores showed some recovery. However, it was rare to observe structural lesions without significant loss of Safranin O staining at some time point in the study, and significant loss of Safranin O staining represents a continued risk for development of structural lesions. Nevertheless, IA injection of TAcs and TA-ER produced very comparable results across all cartilage endpoints. Slight increases in sub-scores at some joint sites occurred with TA-ER compared with TAcs and may be consistent with the longer retention time of TA in the joint. However, inherent variability and the experimental constraints described above must also be considered. Therefore, small differences in scores between groups may not be clinically meaningful in the context of treatment of OA. The Q1M dosing regimen appeared to result in a more favorable long-term outcome with TA-ER when compared with Q3M dosing. However, the different profiles of SF TA exposure suggest that lower drug levels $(<100 \mathrm{ng} / \mathrm{ml})$ during the last month of the interdose interval for the Q3M regimen were probably essential for limiting the extent of cartilage injury in the normal knee.

\section{Intra-Articular Corticosteroids in Normal and Diseased Joints}

The consequences of corticosteroid administration on articular cartilage structure differ depending upon the disease state of the joint and the phenotypic characteristics of the articular chondrocyte. In OA, chondrocytes undergo a phenotypic switch due to activation of mechanical stress-induced and inflammationinduced signaling. Chondrocytes adopt a growth-plate hypertrophied chondrocyte phenotype with re-initiation of proliferation and hypertrophic differentiation. This activated state is associated with a high rate of matrix turnover that is under transcriptional regulation by numerous inflammatory mediators that stimulate matrix degradation and synthesis. In this activated state, catabolic processes predominate, resulting in a net loss of matrix [46-50]. This phenotypic switch in the articular chondrocyte and associated cartilage degeneration is further stimulated by the secondary inflammatory processes in the synovium and subchondral bone that contribute to progression of OA [20, 51-53].

In vitro studies support that the major pharmacological effects of corticosteroids on cartilage matrix homeostasis can be classified as anti-anabolic and anti-catabolic, with anticatabolic effects occurring at concentrations lower than those that affect matrix synthesis $[54,55]$. In healthy cartilage, matrix turnover is low and although catabolic processes play a role in normal cartilage remodeling, these processes are not in an activated state. In vivo studies in animals in the absence of joint inflammation or instability indicate that the anti-anabolic effects of corticosteroids, such as suppression of collagen II synthesis, predominate in the normal remodeling process leading to progressive matrix degeneration that is dose- and durationdependent [29-31, 56-66]. The effects of steroids in normal joints are evidenced histologically, as shown in these studies, by a reduction in Safranin $O$ staining and fissuring of the articular cartilage surface $[31,64,66]$.

In contrast, the therapeutic benefits of corticosteroids are appreciated in the context of OA, where they dampen matrix degeneration by directly inhibiting the exaggerated catabolic processes (such as increased matrix metalloproteinase expression) and indirectly via broad anti-inflammatory effects that limit inflammation-mediated phenotypic alterations in chondrocytes [54]. The chondroprotective effects of corticosteroids in the context of OA is supported by several studies conducted in OA animal models in a variety of species, including dog, horse, rabbit, guinea pig, and rat $[64,67-74]$, demonstrating a reduction in structural damage to the joint with restoration of matrix biosynthesis and a reduction in proteoglycan loss [75].

Previously, TA-ER has demonstrated protective effects on local tissues in a rat model (Lewis rats) characterized by repeated episodes of peptidoglycan polysaccharide (PGPS)-induced 
synovitis [13]. In this experiment, three flares of localized synovitis were induced over 4 weeks following a single IA injection of various doses of TA-ER, TAcs (dose comparable to high-dose TA-ER plasma exposure), or vehicle-diluent. Histopathologic evaluation showed marked-tosevere inflammation with minimal-to-moderate pannus formation and cartilage damage and mild bone resorption in vehicle-diluent-treated rats; TAcs was associated with a smaller nonsignificant improvement in histologic scores compared to vehicle-diluent controls, whereas TA-ER was associated with significant improvement in histologic scores that approximated normal joint tissues.

The effects of corticosteroids on cartilage matrix integrity are therefore context-dependent; chondroprotective effects are observed in the inflammatory milieu of OA where chondrocytes have undergone an inflammation-mediated phenotypic change and matrix remodeling is exaggerated and imbalanced in favor of degradation. In contrast, in normal healthy cartilage where matrix remodeling is balanced and the quiescent articular chondrocyte phenotype is present, the anti-anabolic effects of corticosteroids may result in chondrotoxicity, as seen in these studies.

\section{Synovial Foreign Body Response}

PLGA is a widely used biocompatible and biodegradable drug carrier. An FBR response to PLGA has been observed in association with various routes of administration and differs somewhat according to the physical characteristics of the microspheres, primarily size [76]. Several extended-release PLGA microspherebased products are given via systemic routes (i.e., subcutaneous, intramuscular, and intravitreal routes) and PLGA is also contained in multiple implantable orthopedic devices for long-term use in the knee joint, including anterior cruciate ligament reconstruction, meniscus repair, and fixation of osteochondral fragments.

Consistent, time- and dose-dependent, mild, focal/multifocal FBR to PLGA microspheres were observed in all studies, whether the microspheres were loaded with TA or were blank, although blank microspheres cleared more quickly. The resulting FBR was restricted to the outermost layer of the synovium, the synovial intima. Given sufficient time, the FBR reversed with only some mild residual secondary effects in the synovium (slight focal fibrosis and inflammatory cell infiltrates) in some groups at the end of the repeat-dose study. These residual effects were minimal in severity and are considered to have little clinical significance in the context of the human OA patient population.

Following a single dose, the FBR was evident at day 4, peaked at approximately 4-6 weeks, and was completely resolved by month 6 (representing a drug-free period of only $\sim 3$ months). In repeat-dose studies, the PLGA effects observed with Q1M dosing were like those seen with Q3M dosing with maximal effect at month 1 . The presence of TA did not appear to consistently influence the nature or severity of the FBR and glucocorticoids are known to exert opposing effects on macrophage function dependent on their concentration [77], relative expression, and signaling through both glucocorticoid and mineralocorticoid receptors on macrophages [78]. Nevertheless, the FBR associated with administration of $75 \mathrm{mg}$ of blank microspheres was comparable to that associated with administration of high-dose TA-ER based on qualitative histopathologic assessment.

Mild Safranin O staining loss and structural lesions in articular cartilage occurred in a few 75-mg blank microsphere-treated knees in the repeat-dose study. PLGA-associated FBR in these animals might lead to release of cytokines and chemokines damaging to cartilage and a role for inflammation has been described in cartilage lesions [19]. However, all animals treated with $75 \mathrm{mg}$ of blank microspheres or $75 \mathrm{mg}$ of microspheres as high-dose TA-ER, for either Q3M or Q1M dosing, experienced very similar inflammation in the synovium at month 1 and only slight differences at month 6 . Other factors may have played a role in development of cartilage lesions in blank microsphere-treated knees, but it is also possible that these very mild, low-incidence effects represent intercurrent pathology, particularly given that one 
untreated knee in a single-dose study demonstrated a structural lesion in the patella at month 6 (score 4). In either case, any signal induced by blank microspheres is undetectable against a background of TA-induced cartilage effects in the normal canine knee.

In summary, the normal canine knee joint constitutes a useful model for studying the effects of corticosteroids in normal articular cartilage and FBR to PLGA microspheres after single and repeat dosing. The limitations of this model for predicting human safety should be recognized however, given the differing responses to corticosteroids in articular cartilage in the normal versus diseased state. Also, the FBR to PLGA microspheres is by nature selflimited and resolves as microspheres fragment within the knee joint and disappear after releasing TA-ER. In addition, the studies described here illustrate varying responses that are dependent on intervals between repeat doses, for both TA-ER and TAcs. Slight differences in effects between TA-ER and TAcs may not be significant in a clinical context and could be driven by differences in PK parameters and joint retention times in this model.

\section{CONCLUSIONS}

These findings suggest that the effects of IA injections of TA-ER are generally transient, and comparable to TAcs responses in cartilage in the normal canine knee joint. The effects described here in the normal joint differ from the beneficial effects observed in various disease models and in OA patients. The use of microspheres to extend the period of exposure of the joint to TA results in an FBR that is mild and shows significant recovery or resolution after drug washout. These data support repeat dosing of IA TA-ER in patients with knee OA at an appropriate interval between doses.

\section{ACKNOWLEDGEMENTS}

Funding. Flexion Therapeutics, Inc. supported this work and was involved in the study conception/design. Authors employed by Flexion Therapeutics, Inc. (Neil Bodick, Toni Williamson, Becca Senter, Scott Kelley) revised the article critically for important intellectual content and approved the final submitted version. Flexion Therapeutics, Inc. also funded the article processing charges. All authors had full access to all of the data in this study and take complete responsibility for the integrity of the data and accuracy of the data analysis.

Authorship. All named authors meet the International Committee of Medical Journal Editors (ICMJE) criteria for authorship for this article, take responsibility for the integrity of the work as a whole, and have given their approval for this version to be published.

Medical Writing and Editorial Assistance. Professional medical writing and editing assistance was provided by Michelle L. Perate, MS (independent consultant, Philadelphia, PA, USA) and Jennifer Jaworski, MS (ApotheCom, Yardley, PA, USA); this support was funded by Flexion Therapeutics, Inc. (Burlington, MA, USA).

Author Contributions. All authors (Neil Bodick, Toni Williamson, Vibeke Strand, Becca Senter, Scott Kelley, Rogely Boyce and Ruth Lightfoot-Dunn) contributed to the study conception/design, revised the paper critically for important intellectual content, and approved the final submitted version; Neil Bodick, Toni Williamson and Becca Senter were responsible for data acquisition; Toni Williamson conducted the PK modeling; and Toni Williamson, Vibeke Strand, Becca Senter, Rogely Boyce and Ruth Lightfoot-Dunn collaborated on data analysis/interpretation. Neil Bodick (nbodick@flexiontherapeutics.com) takes responsibility for the integrity of the work as a whole, from inception to finished article.

Disclosures. Neil Bodick is an employee of and owns stock/stock options in Flexion Therapeutics, Inc. Toni Williamson was an employee of Flexion Therapeutics, Inc. at the time of this research. Vibeke Strand is a consultant for Flexion Therapeutics, Inc., AbbVie, EMD 
Serono, Eupraxia, Novartis, Samumed, Servier, and SKK. Becca Senter is an employee of and owns stock/stock options in Flexion Therapeutics, Inc. Scott Kelley is an employee of and owns stock/stock options in Flexion Therapeutics, Inc. Rogely Boyce is employed by Beechy Ridge ToxPath LLC, which provides consulting services to Flexion Therapeutics, Inc. Ruth Lightfoot-Dunn is employed by Crucial Consulting, Inc., which provides consulting services to Flexion Therapeutics, Inc.

Compliance with Ethics Guidelines. Procedures were performed in accordance with regulations outlined in the US Department of Agriculture Animal Welfare Act [22] and in the Institute for Laboratory Animal Research's Guide for Care and Use of Laboratory Animals [23]. The study protocols were reviewed and approved by an institutional animal care and use committee.

Data Availability. These studies have been completed and their datasets are on file with Flexion Therapeutics.

Open Access. This article is distributed under the terms of the Creative Commons Attribution-NonCommercial 4.0 International License (http://creativecommons.org/licenses/ by-nc/4.0/), which permits any noncommercial use, distribution, and reproduction in any medium, provided you give appropriate credit to the original author(s) and the source, provide a link to the Creative Commons license, and indicate if changes were made.

\section{REFERENCES}

1. Osteoarthritis Research Society International (OARSI). Osteoarthritis: A Serious Disease, Submitted to the U.S. Food and Drug Administration. https://www.oarsi.org/sites/default/files/docs/2016/ oarsi_white_paper_oa_serious_disease_121416_1. pdf. Published 2016. Accessed 25 Jan 2018.

2. Robinson $\mathrm{WH}$, Lepus $\mathrm{CM}$, Wang $\mathrm{Q}$, et al. Low-grade inflammation as a key mediator of the pathogenesis of osteoarthritis. Nat Rev Rheumatol. 2016;12(10):580-92.
3. Juni P, Hari R, Rutjes AW, et al. Intra-articular corticosteroid for knee osteoarthritis. Cochrane Database Syst Rev. 2015(10):CD005328.

4. Derendorf H, Mollmann H, Gruner A, Haack D, Gyselby G. Pharmacokinetics and pharmacodynamics of glucocorticoid suspensions after intraarticular administration. Clin Pharmacol Ther. 1986;39(3):313-7.

5. Habib G, Safia A. The effect of intra-articular injection of betamethasone acetate/betamethasone sodium phosphate on blood glucose levels in controlled diabetic patients with symptomatic osteoarthritis of the knee. Clin Rheumatol. 2009;28(1):85-7.

6. Zhang W, Moskowitz RW, Nuki G, et al. OARSI recommendations for the management of hip and knee osteoarthritis, Part II: OARSI evidence-based, expert consensus guidelines. Osteoarthritis Cartilage. 2008;16(2):137-62.

7. McAlindon TE, LaValley MP, Harvey WF, et al. Effect of intra-articular triamcinolone vs saline on knee cartilage volume and pain in patients with knee osteoarthritis: a randomized clinical trial. JAMA. 2017;317(19):1967-75.

8. Kraus VB, Conaghan PG, Aazami HA, et al. Synovial and systemic pharmacokinetics (PK) of triamcinolone acetonide (TA) following intra-articular (IA) injection of an extended-release microsphere-based formulation (FX006) or standard crystalline suspension in patients with knee osteoarthritis (OA). Osteoarthritis Cartil. 2018;26(1):34-42.

9. Russell SJ, Sala R, Conaghan PG, et al. Triamcinolone acetonide extended-release in patients with osteoarthritis and type 2 diabetes: a randomized, phase 2 study. Rheumatology (Oxford). 2018. (in press).

10. Bodick N, Lufkin J, Willwerth C, et al. An intraarticular, extended-release formulation of triamcinolone acetonide prolongs and amplifies analgesic effect in patients with osteoarthritis of the knee: a randomized clinical trial. J Bone Joint Surg Am. 2015;97(11):877-88.

11. Conaghan PG, Hunter DJ, Cohen SB, et al; FX0062014-008 Participating Investigators. Effects of a single intra-articular injection of a microsphere formulation of triamcinolone acetonide on knee osteoarthritis pain: a double-blinded, randomized, placebo-controlled, multinational study. J Bone Joint Surg Am. 2018;100(8):666-77.

12. Conaghan PG, Cohen SB, Berenbaum F, Lufkin J, Johnson JR, Bodick N. Brief report: a phase IIb trial of a novel extended-release microsphere formulation of triamcinolone acetonide for intraarticular 
injection in knee osteoarthritis. Arthritis Rheumatol. $2018 ; 70(2): 204-11$.

13. Kumar A, Bendele AM, Blanks RC, Bodick N. Sustained efficacy of a single intra-articular dose of FX006 in a rat model of repeated localized knee arthritis. Osteoarthritis Cartilage. 2015;23(1):151-60.

14. Boelsterli UA. Animal models of human disease in drug safety assessment. J Toxicol Sci. 2003;28(3):109-21.

15. Buckwalter JA, Mankin HJ. Articular cartilage: tissue design and chondrocyte-matrix interactions. Instr Course Lect. 1998;47:477-86.

16. Maroudas A, Bayliss MT, Uchitel-Kaushansky N, Schneiderman R, Gilav E. Aggrecan turnover in human articular cartilage: use of aspartic acid racemization as a marker of molecular age. Arch Biochem Biophys. 1998;350(1):61-71.

17. Sophia Fox AJ, Bedi A, Rodeo SA. The basic science of articular cartilage: structure, composition, and function. Sports Health. 2009;1(6):461-8.

18. Vandeweerd JM, Zhao Y, Nisolle JF, et al. Effect of corticosteroids on articular cartilage: have animal studies said everything? Fundam Clin Pharmacol. 2015;29(5):427-38.

19. Goldring MB, Otero M. Inflammation in osteoarthritis. Curr Opin Rheumatol. 2011;23(5):471-8.

20. Martel-Pelletier J, Pelletier JP. New insights into the major pathophysiological processes responsible for the development of osteoarthritis. Semin Arthritis Rheum. 2005;34(6 Suppl 2):6-8.

21. Klocke R, Levasseur K, Kitas GD, Smith JP, Hirsch G. Cartilage turnover and intra-articular corticosteroid injections in knee osteoarthritis. Rheumatol Int. 2018;38(3):455-459.

22. USDA Animal Welfare Act; 9 CFR Parts 1, 2 and 3. Federal Register. Published August 31, 1989.

23. National Research Council (US) Committee for the Update of the Guide for the Care Use of Laboratory Animals. Guide for the care and use of laboratory animals. $8^{\text {th }}$ ed. Washington, DC: National Academies Press; 2011.

24. Kraus VB, Stabler TV, Kong SY, Varju G, McDaniel G. Measurement of synovial fluid volume using urea. Osteoarthritis Cartil. 2007;15(10):1217-20.

25. Derendorf H, Nave R, Drollmann A, Cerasoli F, Wurst W. Relevance of pharmacokinetics and pharmacodynamics of inhaled corticosteroids to asthma. Eur Respir J. 2006;28(5):1042-50.
26. Wilkinson M, Jones BS. Electrophoretic studies of synovial fluid proteins. Ann Rheum Dis. 1964;23:22-9.

27. Mankin HJ, Dorfman H, Lippiello L, Zarins A. Biochemical and metabolic abnormalities in articular cartilage from osteo-arthritic human hips. II. Correlation of morphology with biochemical and metabolic data. J Bone Joint Surg Am. 1971;53(3):523-37.

28. Maxie G. Jubb, Kennedy \& Palmer's pathology of domestic animals. 5th ed. New York: Saunders Ltd.; 2007.

29. Ishikawa K. Effect of intra-articular corticosteroid on the meniscus. A histological and histochemical study in rabbit knees. J Bone Joint Surg Am. 1981;63(1):120-30.

30. Ishikawa K, Ohira T, Sakata H. Effects of intraarticular injection of halopredone diacetate on the articular cartilage of rabbit knees: a comparison with methylprednisolone acetate. Toxicol Appl Pharmacol. 1984;75(3):423-36.

31. Behrens F, Shepard N, Mitchell N. Alterations of rabbit articular cartilage by intra-articular injections of glucocorticoids. J Bone Joint Surg Am. 1975;57(1):70-6.

32. Buckwalter JA. Articular cartilage: injuries and potential for healing. J Orthop Sports Phys Ther. 1998;28(4):192-202.

33. Tiku ML, Sabaawy HE. Cartilage regeneration for treatment of osteoarthritis: a paradigm for nonsurgical intervention. Ther Adv Musculoskelet Dis. 2015;7(3):76-87.

34. Pozzi A, Kim SE, Conrad BP, Horodyski M, Banks SA. Ex vivo pathomechanics of the canine PondNuki model. PLoS One. 2013;8(12):e81383.

35. Horisawa E, Kubota K, Tuboi I, et al. Size-dependency of DL-lactide/glycolide copolymer particulates for intra-articular delivery system on phagocytosis in rat synovium. Pharm Res. 2002;19(2):132-9.

36. Tabata Y, Ikada Y. Effect of the size and surface charge of polymer microspheres on their phagocytosis by macrophage. Biomaterials. 1988;9(4):356-62.

37. Wernecke C, Braun HJ, Dragoo JL. The effect of intra-articular corticosteroids on articular cartilage: a systematic review. Orthop J Sports Med. 2015;3(5):2325967115581163.

38. Kiviranta I, Tammi M, Jurvelin J, Arokoski J, Saamanen AM, Helminen HJ. Articular cartilage 
thickness and glycosaminoglycan distribution in the canine knee joint after strenuous running exercise. Clin Orthop Relat Res. 1992;283:302-8.

39. Kiviranta I, Tammi M, Jurvelin J, Helminen HJ. Topographical variation of glycosaminoglycan content and cartilage thickness in canine knee (stifle) joint cartilage. Application of the microspectrophotometric method. J Anat. 1987;150:265-76.

40. Dowthwaite GP, Bishop JC, Redman SN, et al. The surface of articular cartilage contains a progenitor cell population. J Cell Sci. 2004;117(Pt 6):889-97.

41. Pritzker KP, Gay S, Jimenez SA, et al. Osteoarthritis cartilage histopathology: grading and staging. Osteoarthritis Cartilage. 2006;14(1):13-29.

42. Cook JL, Kuroki K, Visco D, Pelletier JP, Schulz L, Lafeber FP. The OARSI histopathology initiativerecommendations for histological assessments of osteoarthritis in the dog. Osteoarthritis Cartil. 2010;18(Suppl 3):S66-79.

43. Bonde HV, Talman ML, Kofoed H. The area of the tidemark in osteoarthritis-a three-dimensional stereological study in 21 patients. APMIS. 2005;113(5):349-52.

44. Walsh DA, McWilliams DF, Turley MJ, et al. Angiogenesis and nerve growth factor at the osteochondral junction in rheumatoid arthritis and osteoarthritis. Rheumatology (Oxford). 2010;49(10):1852-61.

45. Ostergaard K, Andersen CB, Petersen J, Bendtzen K, Salter DM. Validity of histopathological grading of articular cartilage from osteoarthritic knee joints. Ann Rheum Dis. 1999;58(4):208-13.

46. Cecil DL, Johnson K, Rediske J, Lotz M, Schmidt AM, Terkeltaub R. Inflammation-induced chondrocyte hypertrophy is driven by receptor for advanced glycation end products. J Immunol. 2005;175(12):8296-302.

47. Cecil DL, Appleton CT, Polewski MD, et al. The pattern recognition receptor CD36 is a chondrocyte hypertrophy marker associated with suppression of catabolic responses and promotion of repair responses to inflammatory stimuli. J Immunol. 2009;182(8):5024-31.

48. Goldring MB, Otero M, Plumb DA, et al. Roles of inflammatory and anabolic cytokines in cartilage metabolism: signals and multiple effectors converge upon MMP-13 regulation in osteoarthritis. Eur Cell Mater. 2011;21:202-20.

49. Merz D, Liu R, Johnson K, Terkeltaub R. IL-8/ CXCL8 and growth-related oncogene alpha/CXCL1 induce chondrocyte hypertrophic differentiation. J Immunol. 2003;171(8):4406-15.

50. Pitsillides AA, Beier F. Cartilage biology in osteoarthritis-lessons from developmental biology. Nat Rev Rheumatol. 2011;7(11):654-63.

51. Loeser RF. Aging processes and the development of osteoarthritis. Curr Opin Rheumatol. 2013;25(1):108-13.

52. Samuels J, Krasnokutsky S, Abramson SB. Osteoarthritis: a tale of three tissues. Bull NYU Hosp Jt Dis. 2008;66(3):244-50.

53. Scanzello CR. Pathologic and pathogenic processes in osteoarthritis: the effects of synovitis. HSS J. 2012;8(1):20-2.

54. Richardson DW, Dodge GR. Dose-dependent effects of corticosteroids on the expression of matrix-related genes in normal and cytokine-treated articular chondrocytes. Inflamm Res. 2003;52(1):39-49.

55. Tung JT, Venta PJ, Eberhart SW, Yuzbasiyan-Gurkan V, Alexander L, Caron JP. Effects of anti-arthritis preparations on gene expression and enzyme activity of cyclooxygenase- 2 in cultured equine chondrocytes. Am J Vet Res. 2002;63(8):1134-9.

56. Albano MB, Skroch GP, Ioshii SO, Grahels XS, de Alencar PG, Matias JE. Computerized photocolorimetric analysis of the effects of intraarticular betamethasone on the proteoglycan concentration of leporine knee cartilage matrix: influence of the number of intraarticular injections. Rev Col Bras Cir. 2009;36(3):256-60.

57. Chunekamrai S, Krook LP, Lust G, Maylin GA. Changes in articular cartilage after intra-articular injections of methylprednisolone acetate in horses. Am J Vet Res. 1989;50(10):1733-41.

58. Gogia PP, Brown M, al-Obaidi S. Hydrocortisone and exercise effects on articular cartilage in rats. Arch Phys Med Rehabil. 1993;74(5):463-7.

59. Mankin HJ, Conger KA. The acute effects of intraarticular hydrocortisone on articular cartilage in rabbits. J Bone Joint Surg Am. 1966;48(7):1383-8.

60. Moskowitz RW, Davis W, Sammarco J, Mast W, Chase SW. Experimentally induced corticosteroid arthropathy. Arthritis Rheum. 1970;13(3):263-73.

61. Murray RC, DeBowes RM, Gaughan EM, Zhu CF, Athanasiou KA. The effects of intra-articular methylprednisolone and exercise on the mechanical properties of articular cartilage in the horse. Osteoarthritis Cartil. 1998;6(2):106-14. 
62. Papacrhistou G, Anagnostou S, Katsorhis T. The effect of intraarticular hydrocortisone injection on the articular cartilage of rabbits. Acta Orthop Scand Suppl. 1997;275(sup275):132-4.

63. Salter RB, Gross A, Hall JH. Hydrocortisone arthropathy - an experimental investigation. Can Med Assoc J. 1967;97(8):374-7.

64. Shoemaker RS, Bertone AL, Martin GS, et al. Effects of intra-articular administration of methylprednisolone acetate on normal articular cartilage and on healing of experimentally induced osteochondral defects in horses. Am J Vet Res. 1992;53(8):1446-53.

65. Gibson T, Burry HC, Poswillo D, Glass J. Effect of intra-articular corticosteroid injections on primate cartilage. Ann Rheum Dis. 1977;36(1):74-9.

66. Lutfi AM, Kosel K. Effects of intra-articularly administered corticosteroids and salicylates on the surface structure of articular cartilage. J Anat. 1978;127(Pt 2):393-402.

67. Williams JM, Brandt KD. Triamcinolone hexacetonide protects against fibrillation and osteophyte formation following chemically induced articular cartilage damage. Arthritis Rheum. $1985 ; 28(11): 1267-74$.

68. Pelletier JP, Martel-Pelletier J. Protective effects of corticosteroids on cartilage lesions and osteophyte formation in the Pond-Nuki dog model of osteoarthritis. Arthritis Rheum. 1989;32(2):181-93.

69. Pelletier JP, Mineau F, Raynauld JP, Woessner JF Jr, Gunja-Smith Z, Martel-Pelletier J. Intraarticular injections with methylprednisolone acetate reduce osteoarthritic lesions in parallel with chondrocyte stromelysin synthesis in experimental osteoarthritis. Arthritis Rheum. 1994;37(3):414-23.

70. Pelletier JP, DiBattista JA, Raynauld JP, Wilhelm S, Martel-Pelletier J. The in vivo effects of intraarticular corticosteroid injections on cartilage lesions, stromelysin, interleukin-1, and oncogene protein synthesis in experimental osteoarthritis. Lab Invest. $1995 ; 72(5): 578-86$.

71. Foland JW, McIlwraith CW, Trotter GW, Powers BE, Lamar CH. Effect of betamethasone and exercise on equine carpal joints with osteochondral fragments. Vet Surg. 1994;23(5):369-76.

72. Frisbie DD, Kawcak CE, Trotter GW, Powers BE, Walton RM, McIlwraith CW. Effects of triamcinolone acetonide on an in vivo equine osteochondral fragment exercise model. Equine Vet $\mathrm{J}$. 1997;29(5):349-59.

73. Huebner KD, Shrive NG, Frank CB. Dexamethasone inhibits inflammation and cartilage damage in a new model of post-traumatic osteoarthritis. J Orthop Res. 2014;32(4):566-72.

74. Zhang Z, Wei X, Gao J, et al. Intra-articular injection of cross-linked hyaluronic acid-dexamethasone hydrogel attenuates osteoarthritis: an experimental study in a rat model of osteoarthritis. Int J Mol Sci. 2016;17(4):411.

75. Lu YC, Evans CH, Grodzinsky AJ. Effects of shortterm glucocorticoid treatment on changes in cartilage matrix degradation and chondrocyte gene expression induced by mechanical injury and inflammatory cytokines. Arthritis Res Ther. 2011;13(5):R142.

76. Zandstra J, Hiemstra C, Petersen AH, et al. Microsphere size influences the foreign body reaction. Eur Cell Mater. 2014;28:335-47.

77. Lim HY, Muller N, Herold MJ, van den Brandt J, Reichardt HM. Glucocorticoids exert opposing effects on macrophage function dependent on their concentration. Immunology. 2007;122(1):47-53.

78. Rickard AJ, Young MJ. Corticosteroid receptors, macrophages and cardiovascular disease. J Mol Endocrinol. 2009;42(6):449-59. 